\title{
Equilibrium and Arbitrage in Incomplete Asset Markets with Fixed Prices
}

Citation for published version (APA):

Herings, P. J. J., \& Polemarchakis, H. M. (2002). Equilibrium and Arbitrage in Incomplete Asset Markets with Fixed Prices. Journal of Mathematical Economics, 37, 133-155. https://doi.org/10.1016/S03044068(02)00013-7

Document status and date:

Published: 01/01/2002

DOI:

10.1016/S0304-4068(02)00013-7

Document Version:

Publisher's PDF, also known as Version of record

\section{Please check the document version of this publication:}

- A submitted manuscript is the version of the article upon submission and before peer-review. There can be important differences between the submitted version and the official published version of record.

People interested in the research are advised to contact the author for the final version of the publication, or visit the DOI to the publisher's website.

- The final author version and the galley proof are versions of the publication after peer review.

- The final published version features the final layout of the paper including the volume, issue and page numbers.

Link to publication

\footnotetext{
General rights rights.

- You may freely distribute the URL identifying the publication in the public portal. please follow below link for the End User Agreement:

www.umlib.nl/taverne-license

Take down policy

If you believe that this document breaches copyright please contact us at:

repository@maastrichtuniversity.nl

providing details and we will investigate your claim.
}

Copyright and moral rights for the publications made accessible in the public portal are retained by the authors and/or other copyright owners and it is a condition of accessing publications that users recognise and abide by the legal requirements associated with these

- Users may download and print one copy of any publication from the public portal for the purpose of private study or research.

- You may not further distribute the material or use it for any profit-making activity or commercial gain

If the publication is distributed under the terms of Article $25 \mathrm{fa}$ of the Dutch Copyright Act, indicated by the "Taverne" license above, 


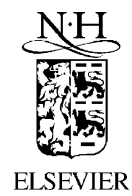

\title{
Equilibrium and arbitrage in incomplete asset markets with fixed prices ${ }^{\text {is }}$
}

\author{
P.J.J. Herings ${ }^{a}, *$, H. Polemarchakis ${ }^{b}$ \\ a Department of Economics, Universiteit Maastricht, P.O. Box 616, \\ 6200 MD Maastricht, The Netherlands \\ ${ }^{\mathrm{b}}$ Department of Economics, Brown University, Providence, RI 02912, USA
}

Received 3 March 2000; received in revised form 18 January 2002; accepted 3 April 2002

\begin{abstract}
At arbitrary prices of commodities and assets, fix-price equilibria exist under weak assumptions: endowments need not satisfy an interiority condition, utility functions need only satisfy a very weak monotonicity requirement, and the asset return matrix allows for redundant assets. Prices of assets may permit arbitrage. At equilibrium, though restricted through endogenously determined trading constraints, arbitrage possibilities may persist; in an example, an individual holds an arbitrage portfolio. () 2002 Elsevier Science B.V. All rights reserved.
\end{abstract}

JEL classification: D45; D52; D60

Keywords: Incomplete asset market; Fix-price equilibrium; Arbitrage

\section{Introduction}

Trade occurs at prices different from competitive equilibrium prices; this is the case in active, competitive markets, asset markets in particular, where prices adjust while purchases and sales are carried out.

The study of markets and the allocations that they generate requires a consistent description of the exchanges that occur at arbitrary prices of commodities and assets. In the market microstructure literature, market makers absorb discrepancies between supply and demand. Here, endogenous bounds on purchases or sales yield market clearing.

\footnotetext{
沦 An earlier version appeared as Discussion Paper No. 0026 (February, 2000), CORE, Université Catholique de Louvain and as METEOR Research Memorandum 00/04, Universiteit Maastricht.

* Corresponding author. Tel.: +31-43-388-3636.

E-mail addresses: p.herings@algec.unimaas.nl (P.J.J. Herings), herakles_polemarchakis@brown.edu (H. Polemarchakis).
} 
The problem that arises is to take the consequences of excess supply and excess demand into account in a way that is consistent both with individual optimization and with the transparency of markets. The definition of a fix-price equilibrium introduced by Bénassy (1975) and Drèze (1975) under certainty extends to economies with uncertainty and an incomplete asset market.

Equilibria exist under extremely weak assumptions. Any assumption on the interiority of individual endowments or on positive aggregate endowments is absent. That minimum wealth is not crucial in models with price rigidities and rationing was observed in Herings (1996); there endogenously emerging constraints might give rise to minimum wealth points. Here, the endowments of individuals may lie on the boundaries of their consumption sets, and the asset market is incomplete; this generality is important in settings with time and uncertainty.

The payoffs of assets are not restricted. With the prices of commodities fixed, assets may be nominal, numeraire or real. More importantly, the asset return matrix need not have full column rank. Redundant assets are allowed, which gives rise to difficulties in the argument for existence, as it is now not trivial to compactify budget sets. To restrict attention to a subset of independent assets is not appropriate: in the presence of trading constraints, an individual may wish to trade in several collinear assets.

The prices of assets may allow for arbitrage. The logical consequence of arbitrage opportunities is that all individuals want to exploit them, and therefore all individuals tend to be on the same side of asset markets that are used to construct an arbitrage portfolio. An individual performing arbitrage will therefore have difficulties in finding trading partners on the other side of the markets. This generates endogenous trading constraints that limit arbitrage opportunities. An important question is whether indeed arbitrage possibilities are completely eliminated by the endogenous trading constraints. Surprisingly, this turns out not to be the case. In an example, an individual holds an arbitrage portfolio at equilibrium, that is supplied, collectively, by the others.

The existence of competitive equilibria was proved by Arrow and Debreu (1954) and McKenzie (1954) in great generality. Crucial to the result, however, was the effective absence of uncertainty. With uncertainty and an incomplete asset market, the existence of competitive equilibria poses important problems.

For the simplest case, with nominal assets, denominated in units of account, or numeraire assets, equilibrium existence results are given in Werner (1985) and Geanakoplos and Polemarchakis (1986). But even in this case, strong convexity and monotonicity assumptions on preferences are not sufficient for the existence of an equilibrium when individual endowments of some commodities are allowed to be zero in some states. Counterexamples to existence were given in Gottardi and Hens (1996). They also provided sufficient conditions for the existence of a competitive equilibrium in the case of numeraire assets, which include strict monotonicity and strict quasi-concavity of the utility function and a strictly positive aggregate endowment as well as a resource relatedness assumption on individual endowments, which strengthens the assumption in McKenzie (1959, 1961). In models with time and uncertainty, even such conditions appear strong, as it is quite likely that in some states of the world certain commodities are not available.

For the case of real assets, a counterexample to existence was given in Hart (1975). A partial rescue of the model relies on the results of Duffie and Shafer (1985), who obtained 
a generic existence result. However, strong differentiability and monotonicity assumptions on utility functions were employed. More importantly, genericity in the payoffs of assets is particularly disturbing; also, for assets whose payoffs are not linear in the prices of commodities, such as options, though Krasa and Werner (1991) obtained generic existence, it is also possible to obtain robust counterexamples to existence, as in Polemarchakis and $\mathrm{Ku}(1990)$.

One can argue that, when a competitive equilibrium does not exist, prices of commodities and assets do emerge against which trade takes place. The determination of such prices would require the specification of a complicated dynamic process. The failure in explaining why prices are rigid and why quantities adjust faster than prices can be considered as a general drawback of the fix-price approach. At least three approaches to explain the rigidity of prices are taken in the fix-price literature. There are models with endogenous price setting of agents with market power, see for instance Bénassy (1988) or Bonanno (1990). Drèze and Gollier (1993) and Drèze (2001) argue that price rigidities are a response to market incompleteness. This argument is particularly valid for the two forms of underemployment of resources most frequently encountered, unemployed labor and excess capacities, two clear examples of commodities for which future markets are hardly developed. Finally, Herings (1997) and Tuinstra (2000) show that political interference in the market mechanism can be rational from a partisan point of view and might be responsible for sustained deviations from prices that clear the markets. Here, we consider the more modest hypothesis that the prices at which trade takes place are given.

The paper is organized as follows. Section 2 introduces the model, the assumptions, and the equilibrium concept. The possible redundancy of the asset return matrix calls for a closer analysis of the set of feasible allocations of assets. In Section 3, the so-called minimal effective feasible allocations of assets are considered, and they are shown to be bounded. Section 4 gives a proof of the existence of equilibrium, and Section 5 illustrates the concepts by analyzing the counterexample to existence of a competitive equilibrium that is given in Hart (1975). Sections 6 and 7 consider the arbitrage opportunities that may be present at equilibrium. Section 6 gives some positive results on the impossibility of performing arbitrage. Section 7 shows the limitations of those results, by means of the example that has been alluded to before.

\section{The economy}

The economy is the standard two-period general equilibrium model with incomplete asset markets and numeraire assets. Transactions occur in assets before and in commodities after the state of nature is known. An economy $\mathcal{E}=\left(\left(\mathcal{X}^{i}, u^{i}, e^{i}\right)_{i \in \mathcal{I}}, R(p, q)\right)$ consists of consumption sets $\mathcal{X}^{i}$, utility functions $u^{i}$ and endowments $e^{i}$ for all individuals $i \in \mathcal{I}$, and an asset return matrix $R(p, q)$ that specifies the payoffs of assets in each state of nature in units of account at prices of commodities $p$ and prices of assets $q$.

States of the world are $s \in \mathcal{S}=\{1, \ldots, \mathcal{S}\}$ and commodities are $l \in \mathcal{L}=\{1, \ldots, L+1\}$. At state $s$, commodity $(L+1, s)$ is assumed to be a numeraire commodity, so its price is $p_{L+1, s}=1$. The domain of prices of commodities is $\mathcal{P}=\left\{p \in \mathbb{R}^{(L+1) S}: p_{L+1, s}=1\right.$, $s \in \mathcal{S}\}$. Assets are $a \in \mathcal{A}=\{1, \ldots, A+1\}$. Asset $A+1$ is assumed to be a numeraire 
asset, its price is $q_{A+1}=1$. The domain of prices of assets is $\mathcal{Q}=\left\{q \in \mathbb{R}^{A+1}: q_{A+1}=1\right\}$. Commodities other than the numeraire are $\breve{\mathcal{L}}=\{1, \ldots, L\}$, and assets other than the numeraire are $\breve{\mathcal{A}}=\{1, \ldots, A\}$.

The numeraire asset plays the role of the medium of exchange before the state of nature is known. After the state of nature has been realized, say the state of nature is $s$, the numeraire commodity $(L+1, s)$ performs this role. Following Geanakoplos and Polemarchakis (1986), it can be shown that a model with first period consumption is a special case of our model.

A utility function $u^{i}$ is weakly monotonically increasing in the numeraire commodity in every state of the world if, for all $x^{i} \in \mathcal{X}^{i}$, for $s \in \mathcal{S}$, for $k \geq 0, u^{i}\left(x^{i}+k 1_{(L+1) s}\right) \geq u^{i}\left(x^{i}\right)$, where $1_{j}$ denotes the $j$-th unit vector of appropriate dimension. Weak monotonicity in the numeraire commodity means that an individual that is given more of the numeraire commodity is not worse off. In particular, it does not exclude noxious non-numeraire commodities or a numeraire commodity that does not enter in the utility function of the individual.

The economy satisfies the following assumptions.

A1. For every individual $i$, the consumption set is $\mathcal{X}^{i}=\mathbb{R}_{+}^{(L+1) S}$.

A2. For every individual $i$, the utility function is continuous, quasi-concave and weakly monotonically increasing in the numeraire commodity in every state of the world.

A3. For every individual $i$, the endowment is an element of the consumption set, $e^{i} \in \mathcal{X}^{i}$.

The endowments are an arbitrary element of the consumption set. No strict positivity assumptions are made. The realistic case that individuals do not possess many commodities or even that some commodities are totally unavailable in certain states of the world is not excluded. This makes it for instance possible to model uncertain outcomes of research and development.

No restrictions are made on the payoffs of assets. Assets may be nominal, numeraire or real. The payoffs of assets may be non-linear in commodity prices, as is the case with options. The asset return matrix need not have full column rank. Redundant assets are allowed for.

Under Assumptions A1-A3, a competitive equilibrium may not exist, as follows from the counterexamples to existence of Hart (1975), Polemarchakis and Ku (1990), and Gottardi and Hens (1996). We take the point of view that even when a competitive equilibrium does not exist, some prices of commodities and assets will emerge against which trade takes place. The explanation of the prices at which trade will eventually take place would require the specification of a complicated dynamic process, which is beyond the scope of the present paper. We start out from the more modest hypothesis that the prices at which trade will take place are given. The challenge is to take into account in a consistent way the consequences of excess supply and excess demand.

We analyse the allocation that results given any terms of trade, that is at any given prices of commodities $\bar{p} \in \mathcal{P}$ and any given prices of assets $\bar{q} \in \mathcal{Q}$. No assumptions are made on $\bar{p}$ and $\bar{q}$, except that they belong to $\mathcal{P}$ and $\mathcal{Q}$. In particular, no non-negativity assumptions are imposed on prices of commodities. Since no monotonicity requirements are imposed on non-numeraire commodities, such non-negativity assumptions would not make sense. 
In certain cases, it might make sense to restrict attention to prices of assets $\bar{q}$ that exclude arbitrage opportunities. Since our analysis is valid for all prices in $\mathcal{Q}$, such an assumption is not made. The asset return matrix at prices $(\bar{p}, \bar{q})$ is $\bar{R}=R(\bar{p}, \bar{q})$.

At arbitrary terms of trade, a competitive equilibrium is typically ruled out. In general, excess supply and excess demand occurs. The specification of an allocation that is consistent with the prices $(\bar{p}, \bar{q})$, with optimizing behavior of individuals, and with transparent markets is non-trivial.

In markets for commodities and assets other than the numeraire, endogenously determined rationing on net trades serves to attain market clearing. To keep the presentation as simple as possible, rationing is assumed to be uniform across individuals. ${ }^{1}$ In case of excess supply in a market, all suppliers will therefore have equal, but limited, opportunities to supply. The limited supply opportunities have spillovers to other markets, which may introduce rationing constraints in markets that cleared before. Rationing in the supply (demand) of commodities other than the numeraire is $\underline{z} \in-\mathbb{R}_{+}^{L s}\left(\bar{z} \in \mathbb{R}_{+}^{L s}\right)$. Rationing in the supply (demand) of assets other than the numeraire is $y \in-\mathbb{R}_{+}^{A}\left(\bar{y} \in \mathbb{R}_{+}^{A}\right)$.

At rationing scheme $(\underline{z}, \bar{z}, \underline{y}, \bar{y})$, the budget set of individual $i$ is

$$
\beta^{i}(\underline{z}, \bar{z}, \underline{y}, \bar{y})=\left(x^{i}, y^{i}\right) \in \mathcal{X}^{i} \times \mathbb{R}^{A+1}:\left\{\begin{array}{l}
\bar{q} y^{i} \leq 0, \\
\bar{p}_{s}\left(x_{s}^{i}-e_{s}^{i}\right) \leq \bar{R}_{s} y^{i}, \quad s \in \mathcal{S}, \\
\underline{y}_{a} \leq y_{a}^{i} \leq \bar{y}_{a}, \quad a \in \check{\mathcal{A}}, \\
\underline{z}_{l, s} \leq x_{l, s}^{i}-e_{l, s}^{i} \leq \bar{z}_{l, s}, \quad(l, s) \in \check{\mathcal{L}} \times \mathcal{S}
\end{array}\right.
$$

The optimization problem of the individual is to choose a utility maximizing consumption bundle and asset portfolio in his budget set. The set of such consumption bundles and asset portfolios is $\delta^{i}(\underline{z}, \bar{z}, \underline{y}, \bar{y})$.

At a given rationing scheme, an individual is effectively rationed in his supply (demand) for a commodity or an asset if he could increase his utility when the rationing scheme in the supply (demand) of that commodity or asset is removed. There is effective supply (demand) rationing in the market for a commodity or an asset if at least one individual is effectively rationed in his supply (demand) for this commodity or asset. Prices $(\bar{p}, \bar{q})$ admit a competitive equilibrium if all markets clear without effective rationing. This makes the concept of competitive equilibrium a special case of the notion here. ${ }^{2}$

Definition 2.1 (Equilibrium). An equilibrium for the economy $\mathcal{E}$ at prices $(\bar{p}, \bar{q})$ is a pair $\left(\left(x^{*}, y^{*}\right),\left(\underline{z}^{*}, \bar{z}^{*}, \underline{y}^{*}, \bar{y}^{*}\right)\right)$ such that

1. for every individual, $\left(x^{i *}, y^{i *}\right) \in \delta^{i}\left(\underline{z}^{*}, \bar{z}^{*}, \underline{y}^{*}, \bar{y}^{*}\right)$,

2. $\sum_{i=1}^{I} x^{i *}=\sum_{i=1}^{I} e^{i}$ and $\sum_{i=1}^{I} y^{i *}=0$,

3. for every $l \in \breve{\mathcal{L}}$, if for some $i^{\prime} x_{l, s}^{i^{\prime} *}-e_{l, s}^{i^{\prime}}=\underline{z}_{l, s}^{*}$, then for all $i \in \mathcal{I} x_{l, s}^{i *}-e_{l, s}^{i}<\bar{z}_{l, s}^{*}$, while if for some $i^{\prime} x_{l, s}^{i^{\prime} *}-e_{l, s}^{i^{\prime}}=\bar{z}_{l, s}^{*}$, then for all $i \in \mathcal{I} x_{l, s}^{i *}-e_{l, s}^{i}>\underline{z}_{l, s}^{*}$, and

\footnotetext{
${ }^{1}$ All our results remain true for more general (non-manipulable) rationing schemes.

${ }^{2}$ When prices are competitive, there might be fix-price equilibrium allocations different from the competitive equilibrium allocation.
} 
4. for every $a \in \check{\mathcal{A}}$, if for some $i^{\prime} y_{a}^{i^{\prime} *}=\underline{y}_{a}^{*}$, then for all $i \in \mathcal{I} y_{a}^{i *}<\bar{y}_{a}^{*}$, while if for some $i^{\prime} y_{a}^{i^{\prime} *}=\bar{y}_{a}^{*}$, then for all $i \in \mathcal{I} y_{a}^{i *}>y_{a}^{*}$.

Conditions 1 and 2 are the usual optimization and market clearing conditions. Conditions 3 and 4, together with the convexity of the consumption sets and the quasi-concavity of the utility functions of individuals, imply that there is no effective rationing, simultaneously, on both sides of a market. This expresses that we do not depart from the scenario of frictionless markets that characterizes competitive equilibria with incomplete markets. Markets are transparent in the sense that it is not possible to find a buyer and a seller in a single market that could benefit from mutual exchange against the numeraire. The definition of equilibrium is a special case of the definition given in Drèze (1975) to analyze the consequences of price rigidities on the allocation of resources in a complete markets setting.

\section{Minimal effective feasible allocations of assets}

The standard approach to show the existence of an equilibrium is to compactify consumption sets, show upper hemi-continuity of the demand correspondence $\delta^{i}, i \in \mathcal{I}$, and apply Kakutani's fixed point theorem to a suitably constructed correspondence. This approach fails in our set-up as a compactified consumption set does not generate bounds on assets portfolios that individuals may be willing to hold. This is due to the absence of an assumption that requires that assets are not collinear.

In the standard incomplete markets model, the presence of collinearity poses no problems. One restricts attention to an independent subset of assets whose span equals the span of the asset return matrix. Such an approach fails in our set-up because endogenous rationing constraints are present. Individuals have good reasons to trade in several collinear assets if this mitigates the restrictions imposed by rationing. There is no way to select an independent subset of assets a priori, without possibly limiting the trading opportunities of the individuals.

In this section, we show that it is still possible to compactify the set of asset portfolios, without reducing the trading opportunities of individuals. Our aim is basically to consider only asset portfolios that are minimally effective, i.e. achieve a certain distribution of revenues over future states with minimal trade in the asset market. A further complication is that one should not consider minimal effective portfolios of assets, but minimal effective feasible allocations of assets. Indeed, if at some given equilibrium $\left(\left(x^{*}, y^{*}\right),\left(z^{*}, \bar{z}^{*}, y^{*}, \bar{y}^{*}\right)\right)$ the asset portfolios $y^{i *}, i \in \mathcal{I}$, are replaced by minimal effective asset portfolios, it is not necessarily the case that the asset markets still clear. Minimal effective feasible allocations of assets is what is called for.

The effective consumption set of individual $i$ is

$$
\overline{\mathcal{X}}^{i}=\left\{x^{i} \in \mathcal{X}^{i}: x_{l, s}^{i} \leq \sum_{i \in \mathcal{I}} e_{l, s}^{i},(l, s) \in \mathcal{L} \times \mathcal{S}\right\} .
$$

If $\left(x^{1}, \ldots, x^{I}\right)$ is a feasible allocation of commodities, then $x^{i} \in \overline{\mathcal{X}}^{i}$ for every individual. Associated with a consumption plan $x^{i}$ of individual $i$, there is a revenue plan 
$w^{i}\left(x^{i}\right)=\left(w_{1}^{i}\left(x_{1}^{i}\right), \ldots, w_{S}^{i}\left(x_{s}^{i}\right)\right)^{\prime} \in \mathbb{R}^{S}$, where $w_{s}^{i}\left(x_{s}^{i}\right)=\bar{p}_{s}\left(x_{s}^{i}-e_{s}^{i}\right)$. The set of effective revenue plans of individual $i$ is

$$
\overline{\mathcal{W}}^{i}=\left\{w^{i} \in \mathbb{R}^{S}: \text { there is } x^{i} \in \overline{\mathcal{X}}^{i} \text { such that } w^{i}=w^{i}\left(x^{i}\right)\right\} .
$$

The set of effective portfolios of assets of individual $i$ is

$$
\overline{\mathcal{Y}}^{i}=\left\{y^{i} \in \mathbb{R}^{A+1}: \bar{q} y^{i}=0, \text { there is } w^{i} \in \overline{\mathcal{W}}^{i} \text { such that } w^{i}=\bar{R} y^{i}\right\} .
$$

The sets $\overline{\mathcal{X}}^{i}$ and $\overline{\mathcal{W}}^{i}$ are obviously compact. This is not necessarily so for the set of effective portfolios of assets of an individual, since the matrix of payoffs of assets need not have full column rank.

The set of effective feasible allocations of assets for the economy is

$$
\overline{\mathcal{Y}}=\left\{y \in \prod_{i \in \mathcal{I}} \overline{\mathcal{Y}}^{i}: \sum_{i \in \mathcal{I}} y^{i}=0\right\} .
$$

Equivalently, $y \in \overline{\mathcal{Y}}$ if there is $w^{i} \in \overline{\mathcal{W}}^{i}, i \in \mathcal{I}$, such that

$$
M y=\left(w^{1^{\prime}}, \ldots, w^{I^{\prime}}, 0,0\right)^{\prime}
$$

where

$$
M=\left[\begin{array}{lll}
\bar{R} & 0 & 0 \\
0 & \ddots & 0 \\
0 & 0 & \bar{R} \\
\bar{q} & 0 & 0 \\
0 & \ddots & 0 \\
0 & 0 & \bar{q} \\
I_{A+1} & \cdots & I_{A+1}
\end{array}\right],
$$

and $I_{A+1}$ denotes the unit matrix of dimension $A+1$. The matrix $M$ is of dimension $(I S+I+A+1) \times I(A+1)$.

The set of minimal effective feasible allocations of assets is

$$
\hat{\mathcal{Y}}=\left\{\bar{y} \in \overline{\mathcal{Y}}: \nexists y \in \overline{\mathcal{Y}} \text { with } \bar{R} y^{i}=\bar{R} \bar{y}^{i}, i \in \mathcal{I}, \operatorname{sign}(y) \in \operatorname{sign}(\bar{y}),|y|<|\bar{y}|\right\},
$$

where $\operatorname{sign}(x)$ denotes the sign vector of $x, \operatorname{Sign}(x)$ a set of sign vectors related to $x$ as specified below, and $|x|$ the absolute value vector associated with the vector $x$. A component of $\operatorname{sign}(x)$ is 1,0 or -1 if the corresponding component of $x$ is $>0,0$ or $<0$, respectively. The set $\operatorname{Sign}(x)$ consists of those sign vectors $v$ for which a component of $v$ is 1 or 0 if the corresponding component of $\operatorname{sign}(x)$ is 1 , a component of $v$ is 0 if the corresponding component of $\operatorname{sign}(x)$ is 0 , and a component of $v$ is -1 or 0 if the corresponding component of $\operatorname{sign}(x)$ is -1 . A component of $|x|$ is the absolute value of the corresponding component of $x$. The set $\hat{\mathcal{Y}}$ contains the effective feasible allocations of assets that are minimal. 
There is no effective feasible allocation of assets such that at least one individual could attain the same revenue plan with less trade, in absolute value, in at least one of the assets.

In the analysis of the set of equilibria of an economy, there is no loss of generality to restrict attention to minimal effective feasible allocations of assets in the following sense. If $\left(\left(x^{*}, y^{*}\right),\left(\underline{z}^{*}, \bar{z}^{*}, \underline{y}^{*}, \bar{y}^{*}\right)\right)$ is an equilibrium for $\mathcal{E}$ at prices $(\bar{p}, \bar{q})$, then there is $\hat{y} \in \hat{\mathcal{Y}}$ such that $\left(\left(x^{*}, \hat{y}\right),\left(\underline{z}^{*}, \bar{z}^{*}, \underline{y}^{*}, \bar{y}^{*}\right)\right)$ is an equilibrium for $\mathcal{E}$ at prices $(\bar{p}, \bar{q})$. Our aim is to show that $\hat{\mathcal{Y}}$ is bounded.

Since $M$ need not have full column rank, the left-inverse of $M$ may not exist. By the singular value decomposition, there exist orthogonal matrices $U$, of dimension $(I(S+1)+$ $A+1) \times(I(S+1)+A+1)$, and $V$, of dimension $I(A+1) \times I(A+1)$, such that $U^{\prime} M V=\Sigma$, where $\Sigma$ is a matrix of dimension $(I(S+1)+A+1) \times I(A+1)$ with non-negative elements $\left(\sigma_{1}, \ldots, \sigma_{I(A+1)}\right)$ on the diagonal and zero off-diagonal elements. ${ }^{3}$ Moreover, there is $r$ such that the first $r$ elements of $\left(\sigma_{1}, \ldots, \sigma_{I(A+1)}\right)$ are positive and the others are zero. The Moore-Penrose inverse of $M$ is defined by $M^{+}=V \Sigma^{+} U^{\prime}$, where $\Sigma^{+}$is a matrix of dimension $I(A+1) \times(I(S+1)+A+1)$ with non-negative elements $\left(1 / \sigma_{1}, \ldots, 1 / \sigma_{r}, 0, \ldots, 0\right)$ on the diagonal and zero off-diagonal elements.

An important property of the Moore-Penrose inverse is the following. Consider some $z \in \mathbb{R}^{I(S+1)+A+1}$. If $y_{R}=M^{+} z$, then $y_{R}$ is an element in the row space of $M$ such that $z=M y_{R}$, and $y_{R}$ is the unique element of the row space of $M$ with this property.

\section{Lemma 3.1. The set $\hat{\mathcal{Y}}$ is bounded.}

Proof. If $\hat{\mathcal{Y}}$ is not bounded, then there exists a sequence $\left(y_{n} \in \hat{\mathcal{Y}}: n=1, \ldots\right)$ such that $\left\|y_{n}\right\|_{\infty} \geq n$. We define $w_{n}=\left(\left(\bar{R} y_{n}^{1}\right)^{\prime}, \ldots,\left(\bar{R} y_{n}^{I}\right)^{\prime}\right)^{\prime}$. Since $\overline{\mathcal{W}}^{i}$ is compact, there is no loss of generality in assuming that the sequence $\left(w_{n} \in \mathbb{R}^{I S}: n=1, \ldots\right)$ is convergent. Moreover, without loss of generality, $\operatorname{sign}\left(y_{n}\right)$ is independent of $n$.

$$
y_{R, n}=M^{+}\left(w_{n}^{\prime}, 0,0\right)^{\prime} \text { and } y_{N, n}=y_{n}-y_{R, n}, \quad \text { for } n=1, \ldots
$$

The sequence $\left(y_{R, n}: n=1, \ldots\right)$ is convergent, and therefore bounded. Since $\left(y_{n} \in \hat{\mathcal{Y}}\right.$ : $n=1, \ldots$ ) is unbounded, without loss of generality, the sequence

$$
\left(\frac{1}{\left\|y_{N, n}\right\|_{\infty}} y_{N, n}: n=1, \ldots\right)
$$

is well-defined and convergent, with limit $\bar{y}_{N}$. Evidently, $M \bar{y}_{N}=0$, and there is $i^{\prime}$ such that $\bar{y}_{N}^{i^{\prime}} \neq 0$.

Moreover, $\bar{y}_{N, a}^{i} \neq 0$ implies $\lim _{n \rightarrow \infty}\left|y_{n, a}^{i}\right|=\infty$, $\operatorname{sign}\left(y_{n, a}^{i}\right)>0$ implies $\bar{y}_{N, a}^{i} \geq 0$, $\operatorname{sign}\left(y_{n, a}^{i}\right)=0$ implies $\bar{y}_{N, a}^{i}=0$, and $\operatorname{sign}\left(y_{n, a}^{i}\right)<0$ implies $\bar{y}_{N, a}^{i} \leq 0$.

So, there exists $n^{\prime}$ such that for $n \geq n^{\prime}, \operatorname{sign}\left(y_{n}-\bar{y}_{N}\right)=\operatorname{sign}\left(y_{n}\right)$.

Furthermore, for $n \geq n^{\prime}, M\left(y_{n}-\bar{y}_{N}\right)=M y_{n}$, whereas $\left|y_{n, a}^{i}-\bar{y}_{N, a}^{i}\right| \leq\left|y_{n, a}^{i}\right|$ and there is $a^{\prime}$ such that $\left|y_{n, a^{\prime}}^{i^{\prime}}-\bar{y}_{N, a^{\prime}}^{i^{\prime}}\right|<\left|y_{n, a^{\prime}}^{i^{\prime}}\right|$.

Hence, for $n \geq n^{\prime}, y_{n} \notin \hat{\mathcal{Y}}$, a contradiction.

\footnotetext{
${ }^{3}$ An orthogonal matrix is a matrix with orthonormal columns, so both $U^{\prime} U$ and $V^{\prime} V$ are identity matrices.
} 
Even when arbitrage possibilities are present, it is possible to restrict attention to a bounded set of asset allocations. Since $\hat{\mathcal{Y}}$ is bounded, there exists $\hat{\alpha}>0$ such that $\|y\|_{\infty}<$ $\hat{\alpha}$ for all $y \in \hat{\mathcal{Y}}$.

\section{The existence of equilibria}

To show the existence of equilibrium, it is essential that budget constraints hold with equality. Since the utility functions of individuals are weakly monotonically increasing in the numeraire commodity, there is no loss of generality in assuming that all second period budget constraints hold with equality. For the first period budget constraint, either one imposes this condition directly on the budget set, or one makes the following assumption.

A4. The numeraire asset satisfies $\bar{R}_{A+1} \geq 0$.

Since the utility functions of individuals are weakly monotonically increasing in all numeraire commodities, $\bar{R}_{\cdot A+1} \geq 0$ implies that the numeraire asset is weakly desirable, so without loss of generality the budget constraint of the individual in the market for assets is satisfied with equality.

It can be verified that if first period consumption is present, assumption A4 is automatically satisfied if the model with first period consumption is rewritten into the one without first period consumption.

At a rationing scheme $(\underline{z}, \bar{z}, \underline{y}, \bar{y})$, the exact budget set $\tilde{\beta}^{i}(\underline{z}, \bar{z}, \underline{y}, \bar{y})$ of individual $i$ is the set of elements $\left(x^{i}, y^{i}\right) \in \beta^{\bar{i}}(\underline{z}, \bar{z}, y, \bar{y})$ that satisfy the budget constraint in every state with equality: $\bar{q} y^{i}=0$ and $\bar{p}_{s}\left(x_{s}^{i}-e_{s}^{i}\right)=\bar{R}_{s} \cdot y^{i}$. The exact demand set $\tilde{\delta}^{i}(\underline{z}, \bar{z}, \underline{y}, \bar{y})$ of the individual is the set of utility maximizing elements $\left(x^{i}, y^{i}\right)$ in $\tilde{\beta}^{i}(\underline{z}, \bar{z}, \underline{y}, \bar{y})$.

Non-emptiness of $\delta^{i}(\underline{z}, \bar{z}, \underline{y}, \bar{y})$ implies non-emptiness of $\tilde{\delta}^{i}(\underline{z}, \bar{z}, \underline{y}, \bar{y})$, since the utility function is weakly monotonically increasing in the numeraire commodity in every state, and because of Assumption A4. Nevertheless, $\tilde{\delta}^{i}(\underline{z}, \bar{z}, \underline{y}, \bar{y})$ can be a proper subset of $\delta^{i}(\underline{z}, \bar{z}, \underline{y}, \bar{y})$, since the utility function is not necessarily strictly monotonically increasing.

Lemma 4.1. If $\mathcal{E}$ satisfies $\mathrm{A} 1-\mathrm{A} 3$, then the correspondence $\tilde{\delta}^{i}$ is non-empty, compact and convex valued, and upper hemi-continuous.

Proof. For any rationing scheme $(\underline{z}, \bar{z}, \underline{y}, \bar{y}),\left(e^{i}, 0\right) \in \tilde{\beta}^{i}(\underline{z}, \bar{z}, \underline{y}, \bar{y})$, so $\tilde{\beta}^{i}(\underline{z}, \bar{z}, \underline{y}, \bar{y})$ is non-empty.

It is obvious that $\tilde{\beta}^{i}(\underline{z}, \bar{z}, \underline{y}, \bar{y})$ is closed and convex.

The set of non-numeraire commodities $\check{\mathcal{L}} \times \mathcal{S}$ is partitioned into the subsets of commodities with positive prices, $\mathcal{K}_{+}$, negative prices, $\mathcal{K}_{-}$, and free commodities, $\mathcal{K}_{0}$. The set of non-numeraire assets $\check{\mathcal{A}}$ is partitioned into the subsets of assets with positive prices, $\mathcal{A}_{+}$, negative prices, $\mathcal{A}_{-}$, and free assets, $\mathcal{A}_{0}$. For $\left(x^{i}, y^{i}\right) \in \tilde{\beta}^{i}(\underline{z}, \bar{z}, \underline{y}, \bar{y}),-\underline{y}_{a} \leq y_{a}^{i} \leq \bar{y}_{a}$, 
$a \in \check{\mathcal{A}}$, and

$$
\begin{aligned}
& y_{A+1}^{i}=-\sum_{a \in \breve{\mathcal{A}}} \bar{q}_{a} y_{a}^{i} \geq-\sum_{a \in \mathcal{A}_{-}} \bar{q}_{a} \underline{y}_{a}-\sum_{a \in \mathcal{A}_{+}} \bar{q}_{a} \bar{y}_{a}, \\
& y_{A+1}^{i}=-\sum_{a \in \check{\mathcal{A}}} \bar{q}_{a} y_{a}^{i} \leq-\sum_{a \in \mathcal{A}_{-}} \bar{q}_{a} \bar{y}_{a}-\sum_{a \in \mathcal{A}_{+}} \bar{q}_{a} \underline{y}_{-},
\end{aligned}
$$

and, thus, the asset demands are bounded. Moreover,

$$
\begin{aligned}
& 0 \leq x_{l, s}^{i} \leq e_{l, s}^{i}+\bar{z}_{l, s}, \quad(l, s) \in \check{\mathcal{L}} \times \mathcal{S}, \\
& 0 \leq x_{L+1, s}^{i} \leq e_{L+1, s}^{i}-\sum_{(l, s) \in \mathcal{K}_{-}} \bar{p}_{l, s} \bar{z}_{l, s}+\sum_{(l, s) \in \mathcal{K}_{+}} \bar{p}_{l, s} e_{l, s}^{i}+\bar{R}_{s} \cdot y^{i}, \quad s \in \mathcal{S},
\end{aligned}
$$

and it follows, from the boundedness of the feasible asset demands, that the feasible spot market demands are bounded as well. Therefore, $\tilde{\beta}^{i}(\underline{z}, \bar{z}, y, \bar{y})$ is compact. By the continuity and quasi-concavity of the utility function, $\tilde{\delta}^{i}(\underline{z}, \bar{z}, y, \bar{y})$ is compact and convex.

Consider a sequence, $\left(\left(\underline{z}_{n}, \bar{z}_{n}, \underline{y}_{n}, \bar{y}_{n}\right) \in-\mathbb{R}_{+}^{L S} \times \mathbb{R}_{+}^{L S} \times-\mathbb{R}_{+}^{A} \times \mathbb{R}_{+}^{A}: n=1, \ldots\right)$ that converges to $(\underline{z}, \bar{z}, \underline{y}, \bar{y})$. For any sequence $\left(\left(x_{n}^{i}, y_{n}^{i}\right) \in \tilde{\delta}^{i}\left(\underline{z}_{n}, \bar{z}_{n}, \underline{y}_{n}, \bar{y}_{n}\right): n=1, \ldots\right)$,

$$
\begin{aligned}
& -\underline{y}_{n, a} \leq y_{n, a}^{i} \leq \bar{y}_{n, a}, \quad a \in \check{\mathcal{A}}, \\
& -\sum_{a \in \mathcal{A}_{-}} \bar{q}_{a} \underline{y}_{n, a}-\sum_{a \in \mathcal{A}_{+}} \bar{q}_{a} \bar{y}_{n, a} \leq y_{n, A+1}^{i} \leq-\sum_{a \in \mathcal{A}_{-}} \bar{q}_{a} \bar{y}_{n, a}-\sum_{a \in \mathcal{A}_{+}} \bar{q}_{a} \underline{y}_{n, a} .
\end{aligned}
$$

Since $\lim _{n \rightarrow \infty}\left(\underline{y}_{n}, \bar{y}_{n}\right)=(\underline{y}, \bar{y})$, it follows that the sequence $\left(y_{n}^{i}: n=1, \ldots\right)$ is bounded. Similarly, since

$$
\begin{aligned}
& 0 \leq x_{n, l, s}^{i} \leq e_{l, s}^{i}+\bar{z}_{n, l, s}, \quad(l, s) \in \check{\mathcal{L}} \times \mathcal{S}, \\
& 0 \leq x_{n, L+1, s}^{i} \leq e_{L+1, s}^{i}-\sum_{(l, s) \in \mathcal{K}_{-}} \bar{p}_{l, s} \bar{z}_{n, l, s}+\sum_{(l, s) \in \mathcal{K}_{+}} \bar{p}_{l, s} e_{l, s}^{i}+\bar{R}_{s} \cdot y_{n}^{i}, \quad s \in \mathcal{S},
\end{aligned}
$$

and since the sequence $\left(\left(\underline{z}_{n}, \bar{z}_{n}\right): n=1, \ldots\right)$ is convergent, the sequence $\left(x_{n}^{i}: n=1, \ldots\right)$ is bounded. It follows that $\left(\left(x_{n}^{i}, y_{n}^{i}\right): n=1, \ldots\right)$ has a convergent subsequence, also denoted $\left(\left(x_{n}^{i}, y_{n}^{i}\right): n=1, \ldots\right)$, with limit $\left(\hat{x}^{i}, \hat{y}^{i}\right) \in \tilde{\beta}^{i}(\underline{z}, \bar{z}, y, \bar{y})$.

If there exists $\left(\tilde{x}^{i}, \tilde{y}^{i}\right) \in \tilde{\delta}^{i}(\underline{z}, \bar{z}, y, \bar{y})$, such that $u^{i}\left(\tilde{x}^{i}\right)>u^{i}\left(\hat{x}^{i}\right)$, then $\tilde{\mathcal{K}}_{-}\left(\tilde{\mathcal{K}}_{+}\right)$is the set of non-numeraire commodities for which $\tilde{x}_{l, s}^{i}-e_{l, s}^{i}$ is negative (positive), and $\tilde{\mathcal{A}}_{-}\left(\tilde{\mathcal{A}}_{+}\right)$is the set of non-numeraire assets for which $\tilde{y}_{a}^{i}$ is negative (positive). Moreover, for $n=1, \ldots$,

$$
\begin{gathered}
\lambda_{n}=\min \left\{1, \frac{\underline{z}_{n, l, s}}{\tilde{x}_{l, s}^{i}-e_{l, s}^{i}},(l, s) \in \tilde{\mathcal{K}}_{-}, \frac{\bar{z}_{n, l, s}}{\tilde{x}_{l, s}^{i}-e_{l, s}^{i}},(l, s) \in \tilde{\mathcal{K}}_{+}, \frac{\underline{y}_{n, a}}{\tilde{y}_{a}^{i}}, a \in \tilde{\mathcal{A}}_{-},\right. \\
\frac{\bar{y}_{n, a}}{\left.\tilde{y}_{a}^{i}, a \in \tilde{\mathcal{A}}_{+}\right\}, \quad \tilde{x}_{n}^{i}=e^{i}+\lambda_{n}\left(\tilde{x}^{i}-e^{i}\right), \quad \tilde{y}_{n}^{i}=\lambda_{n} \tilde{y}^{i} .}
\end{gathered}
$$


Since

$$
\begin{aligned}
& \bar{q} \tilde{y}_{n}^{i}=\lambda_{n} \bar{q} \tilde{y}^{i}=0 \\
& \bar{p}_{s}\left(\tilde{x}_{n, s}^{i}-e_{s}^{i}\right)=\lambda_{n} \bar{p}_{s}\left(\tilde{x}_{s}^{i}-e_{s}^{i}\right)=\lambda_{n} \bar{R}_{s} \tilde{y}^{i}=\bar{R}_{s} \tilde{y}_{n}^{i}, \\
& \tilde{x}_{n, l, s}^{i}-e_{l, s}^{i}=\lambda_{n}\left(\tilde{x}_{l, s}^{i}-e_{l, s}^{i}\right) \geq \frac{\underline{z}_{n, l, s}}{\tilde{x}_{l, s}^{i}-e_{l, s}^{i}}\left(\tilde{x}_{l, s}^{i}-e_{l, s}^{i}\right)=\underline{z}_{n, l, s}, \quad(l, s) \in \tilde{\mathcal{K}}_{-}, \\
& \tilde{x}_{n, l, s}^{i}-e_{l, s}^{i}=\lambda_{n}\left(\tilde{x}_{l, s}^{i}-e_{l, s}^{i}\right) \geq 0 \geq \underline{z}_{n, l, s}, \quad(l, s) \in\left(\check{\mathcal{L}}^{\prime} \times \mathcal{S}\right) \backslash \tilde{\mathcal{K}}_{-}, \\
& \tilde{x}_{n, l, s}^{i}-e_{l, s}^{i}=\lambda_{n}\left(\tilde{x}_{l, s}^{i}-e_{l, s}^{i}\right) \leq \frac{\bar{z}_{n, l, s}}{\tilde{x}_{l, s}^{i}-e_{l, s}^{i}}\left(\tilde{x}_{l, s}^{i}-e_{l, s}^{i}\right)=\bar{z}_{n, l, s}, \quad(l, s) \in \tilde{\mathcal{K}}_{+}, \\
& \tilde{x}_{n, l, s}^{i}-e_{l, s}^{i}=\lambda_{n}\left(\tilde{x}_{l, s}^{i}-e_{l, s}^{i}\right) \leq 0 \leq \bar{z}_{n, l, s}, \quad(l, s) \in(\check{\mathcal{L}} \times \mathcal{S}) \backslash \tilde{\mathcal{K}}_{+}, \\
& \underline{y}_{n, a}=\frac{\underline{y}_{n, a}}{\tilde{y}_{a}^{i}} \tilde{y}_{a}^{i} \leq \lambda_{n} \tilde{y}_{a}^{i}=\tilde{y}_{n, a}^{i} \leq 0 \leq \bar{y}_{n, a}, \quad a \in \tilde{\mathcal{A}}_{-}, \\
& \underline{y}_{n, a} \leq 0 \leq \tilde{y}_{n, a}^{i}=\lambda_{n} \tilde{y}_{a}^{i} \leq \frac{\bar{y}_{n, a}}{\tilde{y}_{a}^{i}} \tilde{y}_{a}^{i}=\bar{y}_{n, a}, \quad a \in \tilde{\mathcal{A}}_{+}, \\
& \left(\tilde{x}_{n}^{i}, \tilde{y}_{n}^{i}\right) \in \tilde{\beta}^{i}\left(\underline{z}_{n}, \bar{z}_{n}, \underline{y}_{n}, \bar{y}_{n}\right) .
\end{aligned}
$$

Evidently, $\lim _{n \rightarrow \infty} \lambda_{n}=1$, and $\lim _{n \rightarrow \infty}\left(\tilde{x}_{n}^{i}, \tilde{y}_{n}^{i}\right)=\left(\tilde{x}^{i}, \tilde{y}^{i}\right)$. By the continuity of the function $u^{i}, u^{i}\left(\tilde{x}_{n}^{i}\right)>u^{i}\left(x_{n}^{i}\right)$ for $n$ sufficiently large, which contradicts $\left(x_{n}^{i}, y_{n}^{i}\right) \in \tilde{\delta}^{i}\left(\underline{z}_{n}, \bar{z}_{n}, \underline{y}_{n}\right.$, $\left.\bar{y}_{n}\right)$. Consequently, $\tilde{\delta}^{i}$ is upper hemi-continuous.

The demand of individuals depends in an upper hemi-continuous way on the constraints they face in the markets of the non-numeraire assets and commodities. It is not necessary to compactify consumption sets in order to get this result, even though there are no restrictions whatsoever in the markets of the numeraire assets and the numeraire commodities.

It is more surprising, and more important, that neither interiority assumptions nor a survival assumption are made with respect to initial endowments. Even though lower hemi-continuity of the budget correspondence in prices may fail, lower hemi-continuity in rationing constraints is satisfied.

The set of equilibria for $\mathcal{E}$ is not compact, because allocations of assets are not necessarily bounded, and rationing schemes are not bounded. There is a compact subset of the set of equilibria that contains all equilibrium allocations.

If $\left(\left(x^{*}, y^{*}\right),\left(z^{*}, \bar{z}^{*}, y^{*}, \bar{y}^{*}\right)\right)$ is an equilibrium for $\mathcal{E}$ at prices $(\bar{p}, \bar{q})$, then there is a minimal effective feasible allocation of assets $\hat{y} \in \hat{\mathcal{Y}}$ satisfying $\sum_{i \in \mathcal{I}} \hat{y}^{i}=0$, and, for every individual, $\bar{R} \hat{y}^{i}=\bar{R} y^{i *}, \bar{q} \hat{y}^{i}=\bar{q} y^{i *}, \operatorname{sign}\left(\hat{y}^{i}\right) \in \operatorname{Sign}\left(y^{i *}\right)$, and $\left|\hat{y}_{a}^{i}\right| \leq\left|y_{a}^{i *}\right|$, for all $a \in \mathcal{A}$. It is not excluded that $\hat{y}=y^{*}$. It follows that $\left(x^{i *}, \hat{y}^{i}\right) \in \tilde{\delta}^{i}\left(\underline{z}^{*}, \bar{z}^{*}, \underline{y}^{*}, \bar{y}^{*}\right), i \in \mathcal{I}$, and that $\left(\left(x^{*}, \hat{y}\right),\left(z^{*}, \bar{z}^{*}, \underline{y}^{*}, \bar{y}^{*}\right)\right)$ is an equilibrium.

In the market for a commodity, $(l, s) \in \check{\mathcal{L}} \times \mathcal{S}$, if there is an individual $i^{\prime}$ such that $x_{l, s}^{i^{\prime} *}-e_{l, s}^{i^{\prime}}=z_{l, s}^{*}$, then by the definition of an equilibrium, no individual is effectively 
rationed in his demand for commodity $(l, s)$, so $x_{l, s}^{i *}-e_{l, s}^{i}<\bar{z}_{l, s}^{*}, i \in \mathcal{I}$. For a fixed $\varepsilon>0$, if $\bar{z}_{l, s}=\varepsilon+\sum_{i \in \mathcal{I}} e_{l, s}^{i}$, then $x_{l, s}^{i *}-e_{l, s}^{i}<\bar{z}_{l, s}, i \in \mathcal{I}$. If there is an individual, $i^{\prime}$, such that $x_{l, s}^{i^{\prime} *}-e_{l, s}^{i^{\prime}}=\bar{z}_{l, s}^{*}$, then no individual is effectively rationed in his supply for commodity $(l, s)$, so $x_{l, s}^{i *}-e_{l, s}^{i}>\underline{z}_{l, s}^{*}, i \in \mathcal{I}$. If $\underline{z}_{l, s}=-\varepsilon-\sum_{i \in \mathcal{I}} e_{l, s}^{i}$, then $x_{l, s}^{i *}-e_{l, s}^{i}>-\underline{z}_{l, s}, i \in \mathcal{I}$.

In the market for some asset $a \in \breve{\mathcal{A}}$, if there is an individual $i^{\prime}$ such that $\hat{y}_{a}^{i^{\prime}}=\underline{y}_{a}$, then no individual is effectively rationed in his demand for asset $a, \hat{y}_{a}^{i}<\bar{y}_{a}^{*}, i \in \mathcal{I}$. Since $\hat{y}_{a}^{i}<\hat{\alpha}$, if $\bar{y}_{a}=\hat{\alpha}$, then $\hat{y}_{a}^{i}<\bar{y}_{a}, i \in \mathcal{I}$. If there is an individual $i^{\prime}$ such that $\hat{y}_{a}^{i^{\prime}}=\bar{y}_{a}^{*}$, then no individual is effectively rationed in his supply for asset $a, \hat{y}_{a}^{i}>y_{a}^{*}, i \in \mathcal{I}$. Since $\hat{y}_{a}^{i}>-\hat{\alpha}$, if $\underline{y}_{a}=-\hat{\alpha}$, then $\hat{y}_{a}^{i}>\underline{y}_{a}, i \in \mathcal{I}$.

In conclusion, if $\left(\left(x^{*}, y^{*}\right),\left(z^{*}, \bar{z}^{*}, \underline{y}^{*}, \bar{y}^{*}\right)\right)$ is an equilibrium, then there is an equilibrium $\left(\left(x^{*}, \hat{y}\right),(\underline{z}, \bar{z}, y, \bar{y})\right)$ with $\|(\underline{z}, \bar{z})\|_{\infty}$ bounded by $\sum_{i \in \mathcal{I}} e^{i}+\varepsilon,\|(y, \bar{y})\|_{\infty}$ bounded by $\hat{\alpha}$, $\left\|x^{*}\right\|_{\infty}$ bounded by $\sum_{i \in \mathcal{I}} e^{i}$ and $\|\hat{y}\|_{\infty}$ bounded by $\hat{\alpha}$. We restrict our attention to rationing schemes and allocations that satisfy these bounds.

The unit cube of dimension $K$ is $\mathcal{C}^{K}=\left\{r \in \mathbb{R}^{K}: 0 \leq r_{k} \leq 1, k=1, \ldots, K\right\}$. The functions $(\underline{z}, \bar{z}): \mathcal{C}^{L S} \rightarrow-\mathbb{R}_{+}^{L S} \times \mathbb{R}_{+}^{L S}$ and $(\underline{y}, \bar{y}): \mathcal{C}^{A} \rightarrow-\mathbb{R}_{+}^{A} \times \mathbb{R}_{+}^{A}$ are defined by

$$
\begin{aligned}
& \underline{z}_{l, s}(r)=-\min \left\{2 r_{l, s}\left(\sum_{i \in \mathcal{I}} e_{l, s}^{i}+\varepsilon\right), \sum_{i \in \mathcal{I}} e_{l, s}^{i}+\varepsilon\right\}, \quad(l, s) \in \check{\mathcal{L}} \times \mathcal{S}, \\
& \bar{z}_{l, s}(r)=\min \left\{\left(2-2 r_{l, s}\right)\left(\sum_{i \in \mathcal{I}} e_{l, s}^{i}+\varepsilon\right), \sum_{i \in \mathcal{I}} e_{l, s}^{i}+\varepsilon\right\}, \quad(l, s) \in \check{\mathcal{L}} \times \mathcal{S}, \\
& \underline{y}_{a}(\rho)=-\min \left\{2 \rho_{a} \hat{\alpha}, \hat{\alpha}\right\}, \quad a \in \check{\mathcal{A}}, \\
& \bar{y}_{a}(\rho)=\min \left\{\left(2-2 \rho_{a}\right) \hat{\alpha}, \hat{\alpha}\right\}, \quad a \in \check{\mathcal{A}},
\end{aligned}
$$

for a fixed $\varepsilon>0$.

Attention is restricted to rationing schemes in the image of the functions $(\underline{z}, \bar{z})$ and $(y, \bar{y})$. The state of the commodity markets is described by $r \in \mathcal{C}^{L S}$ and the state of the asset markets by $\rho \in \mathcal{C}^{A}$. If $0 \leq r_{l, s} \leq 1 / 2$, then there may be supply rationing in the market of commodity $(l, s)$, while demand rationing is excluded by putting $\bar{z}_{l, s}(r)=\sum_{i \in \mathcal{I}} e_{l, s}^{i}+\varepsilon$; if $1 / 2 \leq r_{l, s} \leq 1$, then there may be demand rationing in the market of commodity $(l, s)$, while supply rationing is excluded by putting $\underline{z}_{l, s}(r)=-\sum_{i \in \mathcal{I}} e_{l, s}^{i}-\varepsilon$. If $0 \leq \rho_{a} \leq 1 / 2$, then there may be supply rationing in the market of asset $a$, while demand rationing is excluded by putting $\bar{y}_{a}(\rho)=\hat{\alpha}$; if $1 / 2 \leq \rho_{a} \leq 1$, then there may be demand rationing in the market of asset $a$, while supply rationing is excluded by putting $\underline{y}_{a}(\rho)=-\hat{\alpha}$.

The correspondences $\hat{\delta}^{i}, i \in \mathcal{I}$, and $\hat{\zeta}$, with domain $\mathcal{C}^{L S} \times \mathcal{C}^{A}$ are defined by

$$
\begin{aligned}
& \hat{\delta}^{i}(r, \rho)=\tilde{\delta}^{i}(\underline{z}(r), \bar{z}(r), \underline{y}(\rho), \bar{y}(\rho)), \\
& \hat{\zeta}(r, \rho)=\sum_{i \in \mathcal{I}} \hat{\delta}^{i}(r, \rho)-\left\{\sum_{i \in \mathcal{I}} e^{i}, 0\right\} .
\end{aligned}
$$


The correspondence $\hat{\delta}^{i}$ is a restriction of the correspondence $\tilde{\delta}^{i}$, with rationing schemes being parametrized by the sets $\mathcal{C}^{L S}$ and $\mathcal{C}^{A}$.

Lemma 4.2. If $\mathcal{E}$ satisfies $\mathrm{A} 1-\mathrm{A} 4$, then, if $0 \in \hat{\zeta}\left(r^{*}, \rho^{*}\right)$, there exists $\left(x^{i *}, \hat{y}^{i}\right) \in \hat{\delta}^{i}\left(r^{*}, \rho^{*}\right)$, $i \in \mathcal{I}$, such that $\hat{y} \in \hat{Y}$ and $\left(\left(x^{*}, \hat{y}\right),\left(\underline{z}\left(r^{*}\right), \bar{z}\left(r^{*}\right), \underline{y}\left(\rho^{*}\right), \bar{y}\left(\rho^{*}\right)\right)\right)$ is an equilibrium for $\mathcal{E}$. If $\left(\left(x^{*}, y^{*}\right),\left(z^{*}, \bar{z}^{*}, y^{*}, \bar{y}^{*}\right)\right)$ is an equilibrium for $\mathcal{E}$, then there exists $\left(r^{*}, \rho^{*}\right) \in \mathcal{C}^{L S} \times \mathcal{C}^{A}$ such that $0 \in \hat{\zeta}\left(r^{*}, \rho^{*}\right)$ and there exists $\hat{y} \in \hat{\mathcal{Y}}$ such that $\left(x^{i *}, \hat{y}^{i}\right) \in \hat{\delta}^{i}\left(r^{*}, \rho^{*}\right), i \in \mathcal{I}$.

Proof. If $\left(r^{*}, \rho^{*}\right) \in \mathcal{C}^{L S} \times \mathcal{C}^{A}$ is such that $0 \in \hat{\zeta}\left(r^{*}, \rho^{*}\right)$, then there exists $\left(x^{i *}, y^{i}\right) \in$ $\tilde{\delta}^{i}\left(\underline{z}\left(r^{*}\right), \bar{z}\left(r^{*}\right), \underline{y}\left(\rho^{*}\right), \bar{y}\left(\rho^{*}\right)\right), i \in \mathcal{I}$, such that $\sum_{i \in \mathcal{I}} x^{i *}=\sum_{i \in \mathcal{I}} e^{i}$ and $\sum_{i \in \mathcal{I}} y^{i}=0$. There is a minimal effective feasible allocation of assets $\hat{y} \in \hat{\mathcal{Y}}$, such that $\sum_{i \in \mathcal{I}} \hat{y}^{i}=0$ and, for every individual, $\bar{R} \hat{y}^{i}=\bar{R} y^{i}, \bar{q} \hat{y}^{i}=\bar{q} y^{i}, \operatorname{sign}\left(\hat{y}^{i}\right) \in \operatorname{sign}\left(y^{i}\right)$, and $\left|\hat{y}_{a}^{i}\right| \leq\left|y_{a}^{i}\right|$, $a \in \mathcal{A}$. This implies that $\left(x^{i *}, \hat{y}^{i}\right) \in \tilde{\delta}^{i}\left(\underline{z}\left(r^{*}\right), \bar{z}\left(r^{*}\right), y\left(\rho^{*}\right), \bar{y}\left(\rho^{*}\right)\right)$ and that (1) and (2) of the definition of an equilibrium are satisfied by $\left(\left(x^{*}, \overline{\hat{y}}\right),\left(\underline{z}\left(r^{*}\right), \bar{z}\left(r^{*}\right), \underline{y}\left(\rho^{*}\right), \bar{y}\left(\rho^{*}\right)\right)\right)$.

If, for $(l, s) \in \check{\mathcal{L}} \times \mathcal{S}, x_{l, s}^{i^{\prime} *}-e_{l, s}^{i^{\prime}}=\underline{z}_{l, s}\left(r^{*}\right)$ for some $i^{\prime} \in \mathcal{I}$, then $\underline{z}_{l, s}\left(r^{*}\right) \geq-e_{l, s}^{i^{\prime}}>$ $-\sum_{i \in \mathcal{I}} e_{l, s}^{i}-\varepsilon$. So $r_{l, s}^{*}<1 / 2$, and $\bar{z}_{l, s}\left(r^{*}\right)=\sum_{i \in \mathcal{I}} e_{l, s}^{i}+\varepsilon$. It follows that $x_{l, s}^{i *}-e_{l, s}^{i}<$ $\bar{z}_{l, s}\left(r^{*}\right)$, for every individual.

If, for $(l, s) \in \check{\mathcal{L}} \times \mathcal{S}, x_{l, s}^{i^{\prime} *}-e_{l, s}^{i^{\prime}}=\bar{z}_{l, s}\left(r^{*}\right)$ for some $i^{\prime} \in \mathcal{I}$, then $\bar{z}_{l, s}\left(r^{*}\right) \leq x_{l, s}^{i^{\prime} *}<$ $\sum_{i \in \mathcal{I}} e_{l, s}^{i}+\varepsilon$. So $r_{l, s}^{*}>1 / 2$, and $\underline{z}_{l, s}\left(r^{*}\right)=-\sum_{i \in \mathcal{I}} e_{l, s}^{i}-\varepsilon$. It follows that $x_{l, s}^{i *}-e_{l, s}^{i}>$ $\underline{z}_{l, s}\left(r^{*}\right)$, for every individual.

If, for $a \in \check{\mathcal{A}}, \hat{y}_{a}^{i^{\prime}}=\underline{y}_{a}\left(\rho^{*}\right)$ for some $i^{\prime} \in \mathcal{I}$, then $\underline{y}_{a}\left(\rho^{*}\right)>-\hat{\alpha}$ since $\hat{y} \in \hat{\mathcal{Y}}$. So $\rho_{l, s}^{*}<1 / 2$, and $\bar{y}_{a}\left(\rho^{*}\right)=\hat{\alpha}$. It follows immediately that $\hat{y}_{a}^{i}<\bar{y}_{a}\left(\rho^{*}\right)$, for every individual.

If, for $a \in \check{\mathcal{A}}, \hat{y}_{a}^{i^{\prime}}=\bar{y}_{a}\left(\rho^{*}\right)$ for some $i^{\prime} \in \mathcal{I}$, then $\underline{y}_{a}\left(\rho^{*}\right)<\hat{\alpha}$ since $\hat{y} \in \hat{\mathcal{Y}}$. So $\rho_{l, s}^{*}>1 / 2$, and $\underline{y}_{a}\left(\rho^{*}\right)=-\hat{\alpha}$. Again, it follows immediately that $\hat{y}_{a}^{i}>\underline{y}_{a}\left(\rho^{*}\right)$, for every individual.

Hence, (3) and (4) are satisfied as well in the definition of an equilibrium.

For the second part of the lemma, one supposes that $\left(\left(x^{*}, y^{*}\right),\left(z^{*}, \bar{z}^{*}, \underline{y}^{*}, \bar{y}^{*}\right)\right)$ is an equilibrium for $\mathcal{E}$. It has been argued in Section 3 that there exists $\hat{y} \in \hat{\mathcal{Y}}$ such that $\left(\left(x^{*}, \hat{y}\right),\left(z^{*}, \bar{z}^{*}, \underline{y}^{*}, \bar{y}^{*}\right)\right)$ is an equilibrium for $\mathcal{E}$, so in particular $\left(x^{i *}, \hat{y}^{i}\right) \in \delta^{i}\left(z^{*}, \bar{z}^{*}\right.$, $\left.y^{*}, \bar{y}^{*}\right), i \in \mathcal{I}$. The equality of supply and demand in all markets implies $\left(x^{i *}, \hat{y}^{i}\right) \in$ $\overline{\tilde{\delta}}^{i}\left(\underline{z}^{*}, \bar{z}^{*}, \underline{y}^{*}, \bar{y}^{*}\right), i \in \mathcal{I}$. If there is effective supply rationing in the market for commodity $(l, s) \in \check{\mathcal{L}} \times \mathcal{S}$, then let $r_{l, s}^{*}$ be such that $\underline{z}_{l, s}\left(r^{*}\right)=\underline{z}_{l, s}^{*}$. If there is effective demand rationing in the market for commodity $(l, s) \in \check{\mathcal{L}} \times \mathcal{S}$, then $r_{l, s}^{*}$ is set so that $\bar{z}_{l, s}\left(r^{*}\right)=\bar{z}_{l, s}^{*}$. For all other commodities $(l, s)$, the ones without effective rationing, $r_{l, s}^{*}=1 / 2$. If there is effective supply rationing in the market for asset $a \in \check{\mathcal{A}}$, then $\rho_{a}^{*}$ is set so that $\underline{z}_{a}\left(\rho^{*}\right)=\underline{z}_{a}^{*}$. If there is effective demand rationing in the market for asset $a \in \check{\mathcal{A}}$, then $\rho_{a}^{*}$ is such that $\bar{z}_{a}\left(\rho^{*}\right)=\bar{z}_{a}^{*}$. For all other assets $a$, the ones without effective rationing, define $\rho_{a}^{*}=1 / 2$. It follows from the construction of the functions $(\underline{z}, \bar{z}, \underline{y}, \bar{y})$ that $\left(x^{i *}, \hat{y}^{i}\right) \in \hat{\delta}^{i}\left(r^{*}, \rho^{*}\right), i \in \mathcal{I}$, so $0 \in \hat{\zeta}\left(r^{*}, \rho^{*}\right)$.

The preparatory work is complete. It remains to show that there exists a zero point of $\hat{\zeta}$ and thereby, an equilibrium. By Lemma 4.2, this implies the existence of an equilibrium 
for $\mathcal{E}$. Moreover, the construction used implies that no equilibrium allocations are lost by restricting attention to zero points of $\hat{\zeta}$. Since there is no rationing in the market of the numeraire asset nor in the market of the numeraire commodities, existence of an equilibrium is not obvious.

Theorem 4.3. If $\mathcal{E}$ satisfies $\mathrm{A} 1-\mathrm{A} 4$, then an equilibrium for $\mathcal{E}$ at prices $(\bar{p}, \bar{q})$ exists.

Proof. The correspondence $\hat{\zeta}$ is non-empty, compact, convex valued and upper hemicontinuous. It follows that the set $\hat{\zeta}\left(\mathcal{C}^{L S} \times \mathcal{C}^{A}\right)$ is compact.

The set $\mathcal{Z} \mathcal{Y}$ is compact, convex, and it contains $\hat{\zeta}\left(\mathcal{C}^{L S} \times \mathcal{C}^{A}\right)$. The correspondence $\mu$ : $\mathcal{Z} \mathcal{Y} \rightarrow \mathcal{C}^{L S} \times \mathcal{C}^{A}$ is defined by

$$
\mu(z, y)=\arg \max \left\{\sum_{(l, s) \in \check{\mathcal{L}} \times \mathcal{S}} r_{l, s} z_{l, s}+\sum_{a \in \breve{\mathcal{A}}} \rho_{a} y_{a}: r \in \mathcal{C}^{L S}, \rho \in \mathcal{C}^{A}\right\} .
$$

The correspondence $\varphi: \mathcal{Z Y} \times \mathcal{C}^{L S} \times \mathcal{C}^{A} \rightarrow \mathcal{Z Y} \times \mathcal{C}^{L S} \times \mathcal{C}^{A}$ is defined by

$$
\varphi(z, y, r, \rho)=\hat{\zeta}(r, \rho) \times \mu(z, y)
$$

It is a non-empty, compact, convex valued, upper hemi-continuous correspondence defined on a non-empty, compact, convex set. By Kakutani's fixed point theorem, $\varphi$ has a fixed point, $\left(z^{*}, y^{*}, r^{*}, \rho^{*}\right)$.

If for some $a \in \breve{\mathcal{A}}, y_{a}^{*}<0$, then by the definition of $\mu, \rho_{a}^{*}=0$, so $y_{a}^{*} \geq 0$, a contradiction. If for some $a \in \check{\mathcal{A}}, y_{a}^{*}>0$, then by the definition of $\mu, \rho_{a}^{*}=1$, so $y_{a}^{*} \leq 0$, a contradiction. Consequently, $y_{a}^{*}=0$, for all $a \in \check{\mathcal{A}}$. Moreover, $y_{A+1}^{*}=-\sum_{a \in \check{\mathcal{A}}} \bar{q}_{a} y_{a}^{*}=0$.

If for some $(l, s) \in \check{\mathcal{L}} \times \mathcal{S}, z_{l, s}^{*}<0$, then by the definition of $\mu, r_{l, s}^{*}=0$, so $z_{l, s}^{*} \geq 0$, a contradiction. If for some $(l, s) \in \check{\mathcal{L}} \times \mathcal{S}, z_{l, s}^{*}>0$, then by the definition of $\mu, r_{l, s}^{*}=1$, so $z_{l, s}^{*} \leq 0$, a contradiction. Consequently, $z_{l, s}^{*}=0$, for all $(l, s) \in \check{\mathcal{L}} \times \mathcal{S}$. Moreover, for every $s \in \mathcal{S}, z_{L+1, s}^{*}=-\sum_{(l, s) \in \check{\mathcal{L}} \times \mathcal{S}} \bar{p}_{l, s} z_{l, s}^{*}+\bar{R}_{s} \cdot y^{*}=0$.

It follows that $0 \in \hat{\zeta}\left(r^{*}, \rho^{*}\right)$, and, hence, an equilibrium exists.

It has been argued before that the conditions under which equilibria exist are very weak. No restrictions are made on the prices of assets and commodities, apart from the requirement that the prices of the numeraire assets and the numeraire commodities are 1 . The prices of assets do not have to satisfy the no-arbitrage conditions. Evidently, if the no-arbitrage condition is violated, one expects that all traders want to operate on the same side of the asset markets that are needed to construct an arbitrage portfolio. If indeed all traders are on the same side of an asset market, then no trade is possible in such an asset, as there are no partners to trade with. Although it is shown in Section 6 that this intuition is not entirely correct, it still indicates why violation of the no-arbitrage condition is not inconsistent with existence of equilibrium. Endogenous bounds on trade that arise because of a lack of trading partners restore the existence of equilibrium. 


\section{Hart's counterexample}

To gain some additional insight into our equilibrium concept, it is fruitful to analyze the counterexample to existence of a competitive equilibrium as presented in Hart (1975). We consider the economy $\mathcal{E}=\left(\left(\mathcal{X}^{i}, u^{i}, e^{i}\right)_{i \in \mathcal{I}}, R(p, q)\right)$ with two commodities $(L=1)$ in each of the two states $(S=2)$, two individuals $(I=2)$ and two assets $(A=1)$. The utility functions of the individuals are given by

$$
u^{i}\left(x^{i}\right)=\left(\frac{1}{2}\right) u_{1}^{i}\left(x_{\cdot 1}^{i}\right)+\left(\frac{1}{2}\right) u_{2}^{i}\left(x_{\cdot 2}^{i}\right),
$$

where

$$
\begin{aligned}
& u_{s}^{1}\left(x_{\cdot s}^{1}\right)=\left(\frac{3}{4}\right) \ln \left(x_{1, s}^{1}\right)+\left(\frac{1}{4}\right) \ln \left(x_{2, s}^{1}\right) \\
& u_{s}^{2}\left(x_{\cdot s}^{2}\right)=\left(\frac{1}{4}\right) \ln \left(x_{1, s}^{2}\right)+\left(\frac{3}{4}\right) \ln \left(x_{2, s}^{2}\right),
\end{aligned}
$$

and endowments are

$$
\begin{aligned}
& e^{1}=\left(e_{.1}^{1}, e_{.2}^{1}\right)=\left(\left(\frac{3}{4}, \frac{3}{4}\right),\left(\frac{1}{4}, \frac{1}{4}\right)\right) \\
& e^{2}=\left(e_{.1}^{2}, e_{.2}^{2}\right)=\left(\left(\frac{1}{4}, \frac{1}{4}\right),\left(\frac{3}{4}, \frac{3}{4}\right)\right) .
\end{aligned}
$$

Each of the two future states occurs with probability $1 / 2$, individual 1 spends $75 \%$ of his total income in each state on commodity 1 , and individual 2 spends $75 \%$ of his total income on commodity 2 . Here, income in a state is the income that results after transactions in the asset markets in the first period. Household 1 has high endowments in state 1 and will try to shuffle income to the other state by appropriate transactions on the asset markets, whereas the reverse holds for agent 2 .

Two assets are traded, the futures for commodities 1 and 2, respectively. The asset return matrix in nominal terms is given by

$$
R(p, q)=\left[\begin{array}{ll}
p_{1,1} & p_{2,1} \\
p_{1,2} & p_{2,2}
\end{array}\right] .
$$

The economy $\mathcal{E}$ has no competitive equilibrium, which follows from the arguments provided by Hart (1975). If, at competitive equilibrium prices $\left(p^{*}, q^{*}\right), R\left(p^{*}, q^{*}\right)$ has full rank, markets are complete; the allocational equivalence with a complete markets equilibrium implies that the equilibrium allocation is given by $\left(x^{1 *}, x^{2 *}\right)=((3 / 4,1 / 4,3 / 4,1 / 4),(1 / 4,3 / 4$, $1 / 4,3 / 4))$. Optimization within each state implies that $p_{1,1}^{*}=p_{2,1}^{*}$ and $p_{1,2}^{*}=p_{2,2}^{*}$. Then the rank of $R\left(p^{*}, q^{*}\right)$ is one, a contradiction to the hypothesis that it has full rank. If $R\left(p^{*}, q^{*}\right)$ has rank one, the no-arbitrage condition on prices of assets implies that it is not possible to transfer income from one state into the other one by trade in assets. After a certain state is realized, the economy is like a standard economy with two commodities. It can be verified that $p_{1,1}=(5 / 3) p_{2,1}$ and $p_{1,2}=(3 / 5) p_{2,2}$ is the only possibility to clear the spot markets. But then $R(p, q)$ has full rank, contradicting our supposition. 
As before, the price of the second commodity is normalized to 1. If markets were complete, then the competitive equilibrium price system for commodities would be given by $\bar{p}=$ $((1,1),(1,1))$. The prices of the futures are then determined by a no-arbitrage condition and equal $\bar{q}=(1,1)$. In the economy $\mathcal{E}$ this does not constitute a competitive equilibrium, as markets are not complete if all commodity prices equal to 1 . Endogenous restrictions on trade emerge at those prices.

The requirement $\bar{q} y^{i}=0$ implies $y_{2}^{i}=-y_{1}^{i}$. The structure of the asset return matrix is such that no income can be transferred from one state into another, and any $y^{i}$ such that $y_{2}^{i}=-y_{1}^{i}$ leads to the same consumption possibilities for an individual in the second period. Market clearing implies $y^{2}=-y^{1}$. Any feasible allocation of assets $\left(\left(y_{1}^{1},-y_{1}^{1}\right),\left(-y_{1}^{1}, y_{1}^{1}\right)\right)$ can be replaced by the minimal effective allocation of assets $((0,0),(0,0)) \in \hat{Y}$. Without effective rationing, the demand for commodities of individual 1 in state 1 is $(9 / 8,3 / 8)$ and in state $2(3 / 8,1 / 8)$. Without effective rationing, the demand for commodities of individual 2 is $((1 / 8,3 / 8),(3 / 8,9 / 8))$. There is excess demand for commodity 1 in state 1 and excess supply for commodity 1 in state 2 , which is also consistent with our observation before that a price of $5 / 3$ for commodity 1 in state 1 and a price of $3 / 5$ for commodity 1 in state 2 is needed to clear the markets. The net demand possibilities of individual 1 for commodity 1 in state 1 are determined by the net supply of individual 2 and equal 1/8. Similarly, the net supply possibilities of individual 2 for commodity 1 in state 2 are determined by the net demand of individual 1 and equal $1 / 8$ as well. An equilibrium for the economy $\mathcal{E}$ at prices $\bar{p}=((1,1),(1,1))$ and $\bar{q}=(1,1)$ is $\left(\left(x^{*}, y^{*}\right),\left(z^{*}, \bar{z}^{*}, y^{*}, \bar{y}^{*}\right)\right)$ such that $x^{* 1}=((7 / 8,5 / 8),(3 / 8,1 / 8)), x^{* 2}=((1 / 8,3 / 8),(5 / 8,7 / 8)), y^{* 1}=(0,0)$, $y^{* 2}=(0,0), \bar{z}_{1,1}^{*}=1 / 8, \underline{z}_{1,2}^{*}=-1 / 8$, and the other components of $\underline{z}^{*}$ and $\bar{z}^{*}$, as well as $y^{*}$ and $\bar{y}^{*}$ are chosen as not to be binding.

Another interesting price system to analyze is the one where period 2 commodity prices equilibrate the markets, given that no trade takes place in the asset markets in the first period. That is, $\bar{p}=((5 / 3,1),(3 / 5,1))$, so

$$
\bar{R}=\left[\begin{array}{cc}
\frac{5}{3} & 1 \\
\frac{3}{5} & 1
\end{array}\right]
$$

Suppose again that $\bar{q}=(1,1)$. The first period budget constraint implies $y_{2}^{i}=-y_{1}^{i}$. If no further constraints on supply and demand are present, then optimization of individual 1 at prices $\bar{p}$ and $\bar{q}$ leads to a demand $x^{1}=((3 / 5,1 / 3),(1,1 / 5))$ for commodities and $y^{1}=(-1,1)$ for assets. The payoffs of assets enable the first individual to transfer income to the second state where he is poor, which is achieved by going short in asset 1 and long in asset 2 . The reverse happens for individual 2 , who has a demand $x^{2}=((1 / 5,1),(1 / 3,3 / 5))$ for commodities and $y^{2}=(1,-1)$ for assets if there is no effective rationing.

The asset markets are effectively complete, but the price for commodity 1 in state 1 is higher than the complete markets competitive equilibrium value, whereas the price for commodity 1 in state 2 is lower. It is not surprising that there is excess supply of commodity 1 in state 1 and excess demand for commodity 1 in state 2 . Supply of commodity 1 in state 2 by individual 2 falls short of demand by individual 1 by a rather large amount, which causes individual 1 to be effectively rationed in his demand for that commodity. As a consequence, 
individual 1 transfers less income to state 2, and therefore supplies less of asset 1 in the first period. It also causes individual 1 to demand more of both commodities in state 1. Supply of commodity 1 in state 1 by individual 2 will be constrained by the demand of individual 1 , but the constraint will not bite too much as as an unconstrained individual 2 is not supplying much of that commodity. If individual 2 takes the constraint on the supply of commodity $(1,1)$ into account, his demand for asset 1 will be somewhat smaller than 1 . Since supply of asset 1 by individual 1 is reduced substantially by the prospect of demand rationing in state 2 , individual 2 becomes effectively rationed in his demand for asset 1 . At constraints $\underline{z}_{1,1}^{*}=0$, $\bar{z}_{1,2}^{*}=1 / 3, \bar{y}_{1}^{*}=1 / 2$, the demand of individual 1 is $x^{* 1}=((3 / 4,5 / 12),(7 / 12,1 / 4))$, $y^{* 1}=(-1 / 2,1 / 2)$, and the demand of individual 2 is $x^{* 2}=((1 / 4,7 / 12),(5 / 12,3 / 4))$, $y^{* 2}=(1 / 2,-1 / 2)$ : these constraints indeed induce an equilibrium.

If $\bar{p}=((5 / 3,1),(3 / 5,1))$, then the price for commodity $(1,1)$ is high and the price for commodity $(1,2)$ low relative to a complete markets competitive equilibrium. It is not surprising that in our notion of equilibrium supply rationing arises in the first market and demand rationing in the second. At those prices, individuals utilize the assets to transfer income from one state to another. Even though the prices $\bar{p}=((1,1),(1,1))$ are in accordance with a complete markets competitive equilibrium, whereas the prices $\bar{p}=((5 / 3,1),(3 / 5,1))$ are not, the spanning opportunities offered by the latter, make the equilibrium at those prices Pareto dominate the equilibrium at the former. It can be verified that an increase of the consumption of all commodities in the former equilibrium by $15 \%$ keeps it inferior to the latter.

\section{Arbitrage}

An arbitrage portfolio $\hat{y}$ is such that $\bar{q} \hat{y} \leq 0$, while $\bar{R} \hat{y}>0$. Prices of assets allow for arbitrage if an arbitrage portfolio exists. Theorem 4.3 shows that equilibria exist when prices of assets allow for arbitrage. But the presence of arbitrage opportunities imposes restrictions on rationing in equilibrium.

The utility function of an individual is said to be monotonically increasing in the numeraire commodity at every state of the world if, for all $x^{i} \in \mathcal{X}^{i}$, for $s \in \mathcal{S}$, for $k \geq 0, u^{i}\left(x^{i}+\right.$ $\left.k 1_{(L+1) s}\right)>u^{i}\left(x^{i}\right)$.

Proposition 6.1. If $\mathcal{E}$ satisfies $\mathrm{A} 1-\mathrm{A} 3$ and the utility function of every individual is monotonically increasing in the numeraire commodity at every state of the world, then, if $\left(\left(x^{*}, y^{*}\right)\right.$, $\left.\left(\underline{z}^{*}, \bar{z}^{*}, \underline{y}^{*}, \bar{y}^{*}\right)\right)$ is an equilibrium for $\mathcal{E}$ and $\hat{y}$ is an arbitrage portfolio, there exists for every individual, $i$, an asset, $a \in \check{\mathcal{A}}$, such that either $\hat{y}_{a}<0$ and $y_{a}^{i *}=\underline{y}_{a}^{*}$, or $\hat{y}_{a}>0$ and $y_{a}^{i *}=\bar{y}_{a}^{*}$.

Proof. If the statement is false, then there is an individual, $i$, such that, for every $a \in \check{\mathcal{A}}$, if $\hat{y}_{a}>0, y_{a}^{i *}<\bar{y}_{a}^{*}$, and if $\hat{y}_{a}<0$, then $y_{a}^{i *}>y_{a}^{*}$. It follows that, for some $\lambda>0$, $\underline{y}_{a}^{*} \leq y_{a}^{i *}+\lambda \hat{y}_{a} \leq \bar{y}_{a}^{*}$, for all $a \in \mathcal{A}$. But then, the pair of a consumption plan and a portfolio $\left(x^{i}, y^{i}\right)$ defined by $y^{i}=y^{i *}+\lambda \hat{y}, x_{l, s}^{i}=x_{l, s}^{i *}$, for all $(l, s) \in \check{\mathcal{L}} \times \mathcal{S}$, and $x_{L+1, s}^{i}=x_{L+1, s}^{i *}+\lambda \bar{R}_{s} \hat{y}$, for all $s \in \mathcal{S}$, is an element of the budget set $\beta^{i}\left(\underline{z}^{*}, \bar{z}^{*}, \underline{y}^{*}, \bar{y}^{*}\right)$. 
Since the utility function is monotonically increasing in the numeraire commodity at every state of the world, $u^{i}\left(x^{i}\right)>u^{i}\left(x^{i *}\right)$, a contradiction.

Proposition 6.1 makes precise what sort of endogenous limitations on trade emerge when arbitrage possibilities exist. If arbitrage possibilities are present, then each individual will face constraints on trade in some of the asset markets that are needed to construct an arbitrage portfolio. These constraints are related to the side of the market on which one has to be to perform the arbitrage. The intuition behind this result is clear. If some individual faces no constraints, it would add an arbitrage portfolio to its existing portfolio of assets and thereby increase its utility.

When an arbitrage opportunity is present, all individuals try to profit from it. As a result, it seems likely that all individuals would be on the same side of all asset markets that are used in the arbitrage. The endogenous constraints on trade that emerge would then be such that no trade in these markets is possible.

At an equilibrium, $\left(\left(x^{*}, y^{*}\right),\left(z^{*}, \bar{z}^{*}, y^{*}, \bar{y}^{*}\right)\right)$, the market for asset $a$ is closed if $y_{a}^{*}=0$ or $\bar{y}_{a}^{*}=0$. If a market is not closed, then it is open. In particular, the market for asset $\bar{A}+1$ is always open. The set of all assets for which markets are open is $\mathcal{A}^{\circ}$. The associated effective prices of assets are $q^{\circ}$, an effective portfolio is $y^{\circ}$, and the matrix of effective payoffs of assets is $R^{\circ}$. An effective arbitrage portfolio $\hat{y}^{\circ}$ is such that $q^{\circ} \hat{y}^{\circ} \leq 0$, while $\bar{R}^{\circ} \hat{y}^{\circ}>0$. The intuition of the previous paragraph suggests that effective arbitrage portfolios do not exist.

Proposition 6.2. If $\mathcal{E}$ satisfies $\mathrm{A} 1-\mathrm{A} 3$ and the utility function of every individual is monotonically increasing in the numeraire commodity at every state of the world, then, if $\left(\left(x^{*}, y^{*}\right)\right.$, $\left.\left(z^{*}, \bar{z}^{*}, y^{*}, \bar{y}^{*}\right)\right)$ is an equilibrium for $\mathcal{E}$ at which at most two asset markets are open, $\left|\mathcal{A}^{\circ}\right| \leq \overline{2}$, there is no effective arbitrage portfolio.

Proof. If $\left|\mathcal{A}^{\circ}\right|=1$, the argument is trivial since the only open asset market is the one of the numeraire asset. The existence of an effective arbitrage portfolio is then contradictory to the existence of an equilibrium.

If $\left|\mathcal{A}^{\circ}\right|=2$, then there exists a non-numeraire asset, $\bar{a} \in \mathcal{A}^{\circ}$. If $\hat{y}^{\circ}$ is an effective arbitrage portfolio, then either $\hat{y}_{\bar{a}}^{\circ}=0$ or $\hat{y}_{\bar{a}}^{\circ} \neq 0$. If $\hat{y}_{\bar{a}}^{\circ}=0$, then $q^{\circ} \hat{y}^{\circ} \leq 0$ and $R^{\circ} \hat{y}^{\circ}>0$ implies $R \cdot A+1<0$, so an equilibrium does not exist, a contradiction. If $\hat{y}_{\bar{a}}^{\circ}>0$, then Proposition 6.1 implies that $y_{\bar{a}}^{i *}=\bar{y}_{\bar{a}}^{*}$, for all $i \in \mathcal{I}$. Thus, by market clearing, $\bar{y}_{\bar{a}}^{*}=0$, and the market for asset $\bar{a}$ is not open, a contradiction. If $\hat{y}_{\bar{a}}^{\circ}<0$, it follows by a similar argument that $\underline{y}_{\bar{a}}^{*}=0$, the market for asset $\bar{a}$ is not open, again leading to a contradiction.

Although the result is rather minimal in the sense that it considers only the case with at most two open asset markets, it confirms standard intuition. The existence of effective arbitrage portfolios makes all individuals operate on the same side of the markets involved in the arbitrage, which, as a consequence, close.

\section{An example permitting effective arbitrage portfolios}

The result does not extend to equilibria with three or more open asset markets. With three assets and three individuals, it is even possible that at an equilibrium one individual holds 
an arbitrage portfolio that the other two individuals, together, supply. It is surprising that equilibria with effective arbitrage opportunities may exist.

In the economy $\mathcal{E}=\left(\left(\mathcal{X}^{i}, u^{i}, e^{i}\right)_{i \in \mathcal{I}}, \bar{R}\right)$, there is one commodity $(L=0)$ at each of the three states $(S=3)$, three individuals $(I=3)$ and three assets $(A=2)$. The utility functions are given by

$$
u^{i}\left(x^{i}\right)=a^{i} x_{1}^{i}+b^{i} x_{2}^{i}+c^{i} x_{3}^{i},
$$

where

$$
\begin{aligned}
& \left(a^{1}, b^{1}, c^{1}\right)=(2,1,2), \\
& \left(a^{2}, b^{2}, c^{2}\right)=(1,2,2), \\
& \left(a^{3}, b^{3}, c^{3}\right)=(1,1,2),
\end{aligned}
$$

and endowments are

$$
\begin{aligned}
& e^{1}=\left(e_{1}^{1}, e_{2}^{1}, e_{3}^{1}\right)=(3,9,3), \\
& e^{2}=\left(e_{1}^{2}, e_{2}^{2}, e_{3}^{2}\right)=(9,3,3), \\
& e^{3}=\left(e_{1}^{3}, e_{2}^{3}, e_{3}^{3}\right)=(5,5,5) .
\end{aligned}
$$

Prices of commodities and assets are

$$
\begin{aligned}
& \bar{p}=(1,1,1), \\
& \bar{q}=(1 / 2,1 / 2,1) .
\end{aligned}
$$

The matrix of payoffs of assets is

$$
\bar{R}=\left(\begin{array}{ccc}
-4 & 2 & -2 \\
2 & -4 & -2 \\
2 & 2 & 6
\end{array}\right)
$$

The economy satisfies Assumptions A1-A3, so Propositions 6.1 and 6.2 apply. At an equilibrium with one or two open asset markets, an effective arbitrage portfolio does not exist. Since there are three assets in the economy, Proposition 6.2 does not cover all possible cases.

An arbitrage portfolio $\hat{y}$ satisfies

$$
\begin{aligned}
& -4 \hat{y}_{1}+2 \hat{y}_{2}-2 \hat{y}_{3} \geq 0, \\
& 2 \hat{y}_{1}-4 \hat{y}_{2}-2 \hat{y}_{3} \geq 0, \\
& 2 \hat{y}_{1}+2 \hat{y}_{2}+6 \hat{y}_{3} \geq 0,
\end{aligned}
$$

with at least one strict inequality, and

$$
\hat{y}_{1}+\hat{y}_{2}+2 \hat{y}_{3} \leq 0 \text {. }
$$

For $\lambda>0$, the portfolio $\hat{y}_{\lambda}=(-\lambda,-\lambda, \lambda)$ is an arbitrage portfolio. It holds that $\bar{R} \hat{y}_{\lambda}=$ $(0,0,2 \lambda)^{\prime}>0$, while $\bar{q} \hat{y}_{\lambda}=0$. 
Although the individuals have to choose between three assets and three commodities, using the budget constraints, it is easily seen that they actually face a two-dimensional decision problem. The budget constraint of individual $i$ holds with equality, and $y_{3}^{i}=$ $-(1 / 2) y_{1}^{i}-(1 / 2) y_{2}^{i}$. Since

$$
\bar{R}\left(\begin{array}{c}
y_{1}^{i} \\
y_{2}^{i} \\
-\frac{1}{2} y_{1}^{i}-\frac{1}{2} y_{2}^{i}
\end{array}\right)=\left(\begin{array}{c}
l-3 y_{1}^{i}+3 y_{2}^{i} \\
3 y_{1}^{i}-3 y_{2}^{i} \\
-y_{1}^{i}-y_{2}^{i}
\end{array}\right),
$$

an individual with a utility function $u^{i}\left(x^{i}\right)=a^{i} x_{1}^{i}+b^{i} x_{2}^{i}+c^{i} x_{3}^{i}$ solves the optimization problem

$$
\begin{aligned}
& \max \left(-3 a^{i}+3 b^{i}-c^{i}\right) y_{1}^{i}+\left(3 a^{i}-3 b^{i}-c^{i}\right) y_{2}^{i}, \\
& \text { s.t. } y_{1}^{i}-y_{2}^{i} \leq \frac{1}{3} e_{1}^{i}, \\
& y_{2}^{i}-y_{1}^{i} \leq \frac{1}{3} e_{2}^{i}, \\
& y_{1}^{i}+y_{2}^{i} \leq e_{3}^{i}, \\
& \underline{y}_{1} \leq y_{1}^{i} \leq \bar{y}_{1}, \\
& \underline{y}_{2} \leq y_{2}^{i} \leq \bar{y}_{2} .
\end{aligned}
$$

If $\left(\left(x^{*}, y^{*}\right),\left(\underline{z}^{*}, \bar{z}^{*}, y^{*}, \bar{y}^{*}\right)\right)$ is an equilibrium for $\mathcal{E}$ at prices $(\bar{p}, \bar{q})$, since, for any $\lambda>0$, $y_{\lambda}$ is an arbitrage portfolio, it follows by Proposition 6.1 that all individuals are effectively rationed in the supply of asset 1 or asset 2 . If no individual is effectively rationed in the supply of asset 2 , then every individual is effectively rationed in the supply of asset 1 , and market clearing implies that $y_{1}^{*}=0$. Irrespective of rationing in the demand of asset 2 , individual 2 supplies 2 units of asset 2 and individual 3 supply 4/3 units of asset 2 , whereas individual 1 demands at most 2 units of this asset, which is a contradiction. Similarly, there is no equilibrium without effective rationing in the supply of asset market 1 . Consequently, in every equilibrium, there is effective rationing in the supply of both assets. Condition 4 in the definition of an equilibrium implies that there is no effective rationing in the demand of any asset. Therefore, the demand for assets 1 and 2, and, hence, for asset 3 as well as for commodities, is a function of the rationing scheme on the supplies of the assets. The derivation of the demand functions is facilitated by the graphic illustration of the decision problem of individual $i$ depicted in Fig. 1, where the rationing scheme is taken equal to $y=(-1,-1)$.

It is immediately verified that the situation depicted in Fig. 1 constitutes an equilibrium $\left(\left(x^{*}, y^{*}\right),\left(z^{*}, \bar{z}^{*}, y^{*}, \bar{y}^{*}\right)\right)$, with $z^{*}$ and $\bar{z}^{*}$ not coming into play since there are no non-numeraire commodities, $y^{*}=(-1,-1)^{\prime}, \bar{y}^{*}>(2,2)^{\prime}$ (the exact choice does not matter). Then $x^{1 *}=(12,0,2)^{\prime}, x^{2 *}=(0,12,2)^{\prime}, x^{3 *}=(5,5,7)^{\prime}, y^{1 *}=(-1,2,-1 / 2)^{\prime}$, $y^{2 *}=(2,-1,-1 / 2)^{\prime}$, and $y^{3 *}=(-1,-1,1)^{\prime}$. This describes the unique equilibrium, 


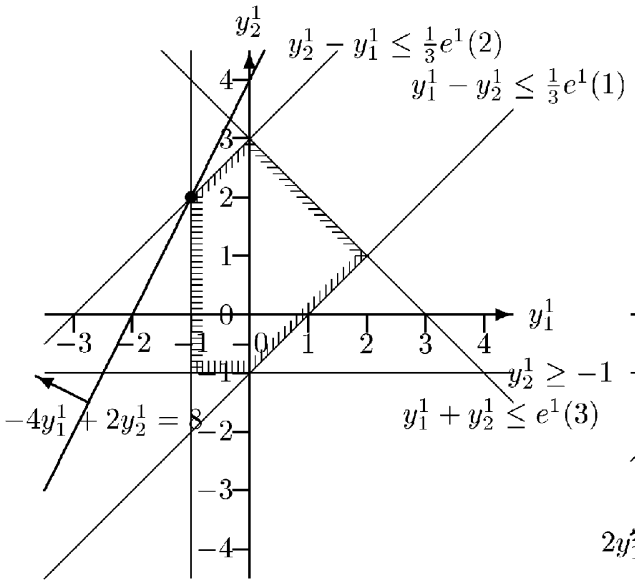

$y_{1}^{1} \geq-1$

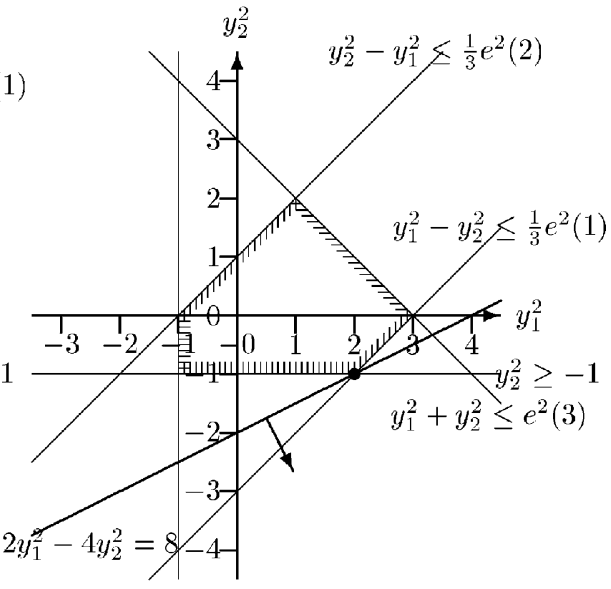

$y_{1}^{2} \geq-1$

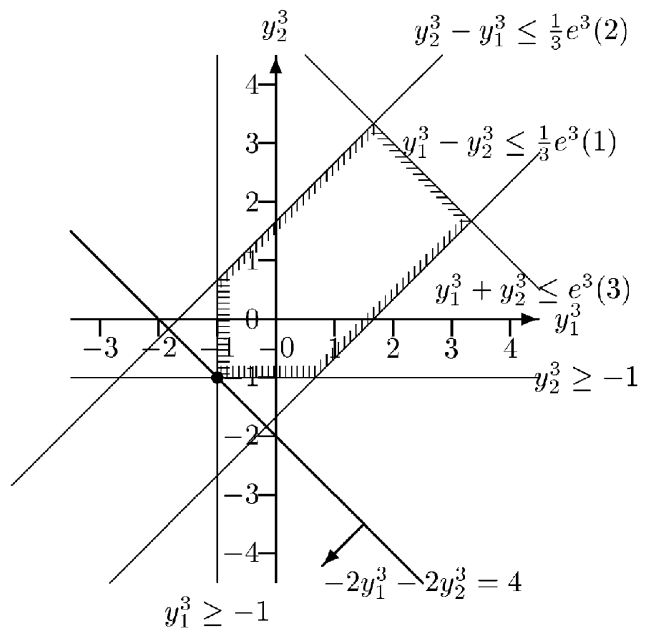

27

Fig. 1. Decision problems of the three individuals.

where equilibria are equivalent if they differ only with respect to rationing schemes that are not effective.

Indeed, the demands of individuals as functions of the rationing scheme on the supplies are

$$
\begin{aligned}
& x^{1}(\underline{y})=\left(12,0, \min \left\{-2 \underline{y}_{1}, 6-2 \underline{y}_{2}\right\}\right)^{\prime}, \\
& y^{1}(\underline{y})=\left(\max \left\{\underline{y}_{1}, \underline{y}_{2}-3\right\}, \max \left\{3+\underline{y}_{1}, \underline{y}_{2}\right\}, \min \left\{-1 \frac{1}{2}-\underline{y}_{1}, 1 \frac{1}{2}-\underline{y}_{2}\right\}\right)^{\prime},
\end{aligned}
$$




$$
\begin{aligned}
& x^{2}(\underline{y})=\left(0,12, \min \left\{-2 \underline{y}_{2}, 6-2 \underline{y}_{1}\right\}\right)^{\prime}, \\
& y^{2}(\underline{y})=\left(\max \left\{3+\underline{y}_{2}, \underline{y}_{1}\right\}, \max \left\{\underline{y}_{2}, \underline{y}_{1}-3\right\}, \min \left\{-1 \frac{1}{2}-\underline{y}_{2}, 1 \frac{1}{2}-\underline{y}_{1}\right\}\right)^{\prime}, \\
& x^{3}(\underline{y})=\left(5-3 \underline{y}_{1}+3 \underline{y}_{2}, 5+3 \underline{y}_{1}-3 \underline{y}_{2}, 5-\underline{y}_{1}-\underline{y}_{2}\right)^{\prime}, \\
& y^{3}(\underline{y})=\left(\underline{y}_{1}, \underline{y}_{2},-\frac{1}{2} \underline{y}_{1}-\frac{1}{2} \underline{y}_{2}\right)^{\prime} .
\end{aligned}
$$

The equality of supply and demand for assets 1 and 2 , necessary and sufficient for equilibrium, yields

$$
\begin{aligned}
& \max \left\{\underline{y}_{1}, \underline{y}_{2}-3\right\}+\max \left\{3+\underline{y}_{2}, \underline{y}_{1}\right\}+\underline{y}_{1}=0, \\
& \max \left\{3+\underline{y}_{1}, \underline{y}_{2}\right\}+\max \left\{\underline{y}_{2}, \underline{y}_{1}-3\right\}+\underline{y}_{2}=0 .
\end{aligned}
$$

The unique solution is $y=(-1,-1)^{\prime}$.

At the equilibrium, individuals 1 and 2, together, supply the arbitrage portfolio that individual 3 holds.

\section{Conclusion}

At any prices for commodities and assets, with rationing, an equilibrium allocation of resources exists under weak assumptions. There is no need to resort to a generic argument, even when markets are incomplete and assets are real or display an even more complicated dependence on prices. Neither is there a need to make the usual, but unappealing interiority assumption on endowments.

The equilibrium concept also provides a solution when the no-arbitrage condition on prices is not satisfied. The logical consequence of the existence of arbitrage portfolios is that all individuals try to exploit these arbitrage opportunities. This limits the possibilities to find trading partners needed for the arbitrage, which generates endogenously determined constraints on such trades. Even though markets clear in our concept of equilibrium, market clearance generally involves endogenously determined amounts of effective rationing.

Even though arbitrage possibilities are limited by endogenously generated constraints on trade, it is not necessarily the case that all arbitrage opportunities are eliminated. It is even possible for an individual to hold an arbitrage portfolio in equilibrium, which is, because of market clearing, supplied by others. This phenomenon is rather counterintuitive since the other individuals are not excluded from holding the arbitrage portfolio themselves.

\section{Acknowledgements}

The research of Herings was made possible by a fellowship of the Royal Netherlands Academy of Arts and Sciences and a grant of The Netherlands Organization for Scientific Research (NWO); while this paper was being written, he enjoyed the generous hospitality of the Cowles Foundation. 


\section{References}

Arrow, K.J., Debreu, G., 1954. Existence of an equilibrium for a competitive economy. Econometrica 22, 265-290. Bénassy, J.-P., 1975. Neo-Keynesian disequilibrium theory in a monetary economy. Review of Economic Studies 42, 503-523.

Bénassy, J.-P., 1988. The objective demand curve in general equilibrium with price makers. Economic Journal 98, 37-49.

Bonanno, G., 1990. General equilibrium theory with imperfect competition. Journal of Economic Surveys 4, 297-328.

Drèze, J.H., 1975. Existence of an exchange equilibrium under price rigidities. International Economic Review $16,301-320$.

Drèze, J.H., 2001. On the macroeconomics of uncertainty and incomplete markets. Recherches Economiques de Louvain 67, 5-30.

Drèze, J.H., Gollier, C., 1993. Risk sharing on the labour market. European Economic Review 37, 1457-1482.

Duffie, D., Shafer, W., 1985. Equilibrium in incomplete markets. I. A basic model of generic existence. Journal of Mathematical Economics 14, 285-300.

Geanakoplos, J.D., Polemarchakis, H.M., 1986. Existence, regularity, and constrained suboptimality of competitive allocations when the asset market is incomplete. In: Heller, W.P., Starr, R.M., Starrett, D.A. (Eds.), Uncertainty, Information and Communication: Essays in Honor of K.J. Arrow, Vol. 3. Cambridge University Press, Cambridge, pp. 65-96.

Gottardi, P., Hens, T., 1996. The survival assumption and existence of competitive equilibria when asset markets are incomplete. Journal of Economic Theory 71, 313-323.

Hart, O.D., 1975. On the optimality of equilibrium when the market structure is incomplete. Journal of Economic Theory 11, 418-443.

Herings, P.J.J., 1996. Equilibrium existence results for economies with price rigidities. Economic Theory 7, 63-80.

Krasa, S., Werner, J., 1991. Equilibria with options: existence and indeterminacy. Journal of Economic Theory 54, 305-320.

McKenzie, L., 1954. On equilibrium in Graham's model of world trade and other competitive systems. Econometrica 22, 147-161.

McKenzie, L., 1959. On the existence of general equilibrium for a competitive economy. Econometrica 27, 54-71.

McKenzie, L., 1961. On the existence of general equilibrium: some corrections. Econometrica 29, 247-248.

Polemarchakis, H.M., Ku, B., 1990. Options and equilibrium. Journal of Mathematical Economics 19, $107-112$.

Tuinstra, J., 2000. The emergence of political business cycles in a two-sector general equilibrium model. European Journal of Political Economy 16, 509-534.

Werner, J., 1985. Equilibrium in economies with incomplete financial markets. Journal of Economic Theory 36, 110-119. 